\title{
Extrasynaptic GABA waves underlie epileptiform rhythms in the hippocampal network
}

Vincent Magloire ${ }^{1} \#^{*}$, Leonid P. Savtchenko ${ }^{1} \#^{*}$, Sergyi Sylantyev ${ }^{1,2}$, Thomas P. Jensen ${ }^{1}$, Nicholas Cole ${ }^{1}$, Jonathan S. Marvin³ ${ }^{3}$ Loren L. Looger ${ }^{3}$, Dimitri M. Kullmann', Matthew C. Walker ${ }^{1}$, Ivan Pavlov#, Dmitri A. Rusakov#

${ }^{1}$ UCL Queen Square Institute of Neurology, University College London, Queen Square, London WC1N 3BG, UK

${ }^{2}$ Rowett Institute, University of Aberdeen, Ashgrove Rd. West, Aberdeen AB25 2ZD, UK

${ }^{3}$ Janelia Research Campus, Howard Hughes Medical Institute, Ashburn, Virginia, USA

*- equal contributions

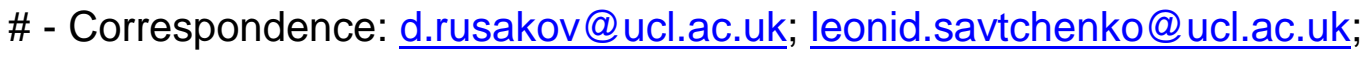
v.magloire@ucl.ac.uk; i.y.pavlov@gmail.com; 


\begin{abstract}
The cellular and circuit mechanisms that trigger and maintain epileptiform discharges remain the subject of intense debate. We have earlier reported a bell-shape dependence of spiking activity of interneuronal populations on tonic $\mathrm{GABA}_{\mathrm{A}}$ receptor conductance (Gtonic), suggesting an innate mechanism to enable slow self-sustained network oscillations. In the brain, Gtonic is controlled by the slow changes of the extracellular GABA concentration ([GABA]e), which in turn depends on spiking activity of interneurons. Here, we employ outside-out patch-clamp recordings of GABAA receptor and fluorescence imaging of a GABA sensor to show that periodic epileptiform discharges are preceded by [GABA] rises. Computer simulations of spiking interneuronal networks reveal that incorporating extrasynaptic waves of

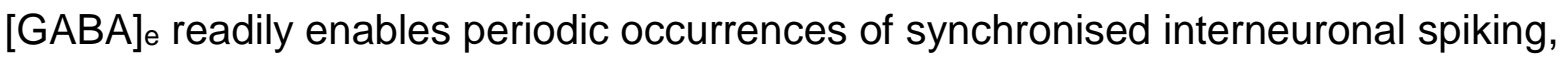
which are in phase with rises in Gtonic and can trigger short bursts of principal cell spiking. Simultaneous recording from multiple neurons and selective optogenetic stimulation of parvalbumin-positive $(\mathrm{PV}+)$ interneurons confirmed the modelling predictions, consistent with a causal relationship between synchronisation of interneuronal activity, inhibitory synaptic input, Gtonic, and interictal events. Our findings suggest a key role of [GABA] dynamics in enabling and pacing regenerative rhythmic activity of brain networks.
\end{abstract}




\section{Introduction}

GABA is the principal inhibitory neurotransmitter in the forebrain. It mediates conventional fast synaptic transmission through $G A B A_{A}$ receptors ( $G A B A_{A} R s$ ), as well as slower synaptic transmission mediated by $G A B A_{B}$ receptors. There is also a slow $\mathrm{GABA}_{A} \mathrm{R}$-mediated conductance (Gtonic), often referred to as 'tonic inhibition', which reflects activation of slowly desensitizing, extrasynaptic GABAARs by GABA that is continuously present in the extracellular space. Historically, micro-dialysis studies in vivo have suggested that the ambient extracellular GABA concentration ([GABA]e) is relatively stable, even though it may depend on the physiological state and can change with exploratory behaviour (reviewed in ${ }^{1}$ ). Indeed, extrasynaptic GABA escape is constrained by GABA transporters ${ }^{2,3}$, which should curtail large changes in $[\mathrm{GABA}]_{\mathrm{e}}$. However, the dialysis technique usually reports [GABA] averaged over tens of seconds whereas the relatively low apparent affinity $\left(K_{m}=10-20 \mu \mathrm{M}\right)$ of the main GABA transporter GAT-1 ${ }^{4-6}$ cannot prevent faster, low micromolar-range fluctuations in activity-dependent [GABA]e.

The dynamics of Gtonic has been associated with the firing pattern of interneurons ${ }^{7}$ and implicated, at least theoretically, in epileptiform discharge initiation 3,8 . Activation of extrasynaptic GABA $\mathrm{A}_{\mathrm{R}}$ can also alter the excitability of principal cells ${ }^{9-12}$, which directly influences network behaviour ${ }^{13,14}$ including input coincidence detection rules 15-17. Whilst a collapse in inhibitory activity has been associated with the emergence of epileptiform events ${ }^{18}$, hippocampal interneurons appear to maintain or increase their firing rate before the onset of ictal activity ${ }^{19}$. In the cortex, seizure-like events in pyramidal neurons were preceded by an outward $\mathrm{GABA}_{A} \mathrm{R}$ current and a rises in extracellular $\mathrm{K}^{+20}$ whereas optogenetic activation of GABAergic cells en masse could by itself trigger ictal events as post-inhibitory excitation sprung back ${ }^{21}$. While these observations clearly implicate dynamic interactions between network inhibition and disinhibition (or rebound excitation) in the generation of ictal discharges, the precise nature of these interactions and their mechanistic role in maintaining rhythmic epileptiform activity remains poorly understood.

Intriguingly, we have previously found a biphasic, bell-shaped relationship between Gtonic (controlled through either dynamic clamp or by changing ambient [GABA] $)$, and the firing rate and synchronisation in pharmacologically isolated interneuronal networks ${ }^{14}$. Because Gtonic itself depends on interneuronal firing, this type of relationship suggests, at least in theory, an inherent capacity of self-organising rhythms, or regular waves of activity ${ }^{22}$. Genetically encoded optical GABA sensors have recently enabled the detection of prominent [GABA] transients (on a 10-100 ms timescale) during synchronous ictal discharges in vivo ${ }^{23}$, arguing that fluctuations in Gtonic may have a role in periodic network activity in the intact brain.

We therefore sought to understand how the dynamics of [GABA] $]_{e}$ and Gtonic both arise from and control the firing activity of neuronal networks, leading to its rhythmic patterns. To this end, we focused on FS hippocampal interneurons and tested the hypothesis that their synchronized firing should trigger transient changes in [GABA] that help maintain sustained network rhythms, such as inter-ictal epileptiform 
discharges. We therefore combined outside-out membrane patch (GABA sniffer) recordings ${ }^{24,25}$, an optical GABA sensor iGABASnFR ${ }^{23}$, and optogenetic stimulation of PV-positive interneurons, with computer simulations of spiking neural networks 26,27 , to establish the role of [GABA] e waves as a powerful feedback mechanism that paces cortical network oscillations.

\section{RESULTS}

\section{Transient $[\mathrm{GABA}]_{\mathrm{e}}$ rises in tissue tend to precede interictal events}

To examine the relationship between regular bursts of neuronal firing and [GABA]e, we used acute hippocampal slices perfused with a solution containing $0\left[\mathrm{Mg}^{2+}\right]$ and $5 \mathrm{mM}$ $\left[\mathrm{K}^{+}\right]$, in order to elicit epileptiform activity. In the CA1 region, this activity was detected as brief (<200 ms) periodic population bursts (Fig. 1a, top trace). In parallel, we monitored local [GABA] e using an outside-out 'sniffer' patch which reports $\mathrm{GABA}_{A} \mathrm{R}$ channel activity ${ }^{24}$, as we previously demonstrated ${ }^{17,25}$ (Fig. 1a, middle; Methods). The channel opening activity in the patch (Fig. 1a, bottom) displayed a clear temporal correlation with field potential bursts (Fig. 1a-c). A detailed analysis revealed that a transient increase in this activity, hence in [GABA] e, consistently started $0.5-1 \mathrm{~s}$ before interictal bursts (summary Fig. 1d, also examples in Fig. 1b-c). We took special care to prepare uniformly shaped sniffer patch pipettes for these experiments, and used a multi-channel rapid solution exchange system ${ }^{28}$ to estimate $\mathrm{EC}_{50}$ of [GABA] for patch recordings: the average $\mathrm{EC}_{50}$ value was $\sim 1.1 \mu \mathrm{M}$, which corresponds to a charge transfer rate (current) of $256 \pm 57 \mathrm{fC} \cdot \mathrm{s}^{-1}(\mathrm{f} A ; n=6$; Fig. 1e). During interictal events, the sniffer-patch detected [GABA] e transients from a resting level of $\sim 0.3 \mu \mathrm{M}$ peaking at $1.5-2 \mu \mathrm{M}$.

We next explored the cellular basis of these observations. In these settings, the spiking activity of individual FS PV+ interneurons increased substantially from their basal level several seconds before the peak discharge of CA1 pyramidal cells (which correspond to the interictal events, Extended Data Fig. 1a-b). This was faithfully reflected by the elevated inhibitory input to CA1 pyramidal cells before the interictal bursts (Extended Data Fig. 1c-d). In contrast, pyramidal cell spiking appeared timelocked to the epileptiform discharges (Extended Data Fig. 1e-f). These observations indicate that interictal bursting is preceded, somewhat paradoxically, by increased firing of FS PV+ interneurons and increased GABAergic receptor current (as noted earlier ${ }^{20,21}$ ), which is paralleled by the rise in [GABA] detected with the 'sniffer' patch (Fig. 1).

\section{Interictal spikes are preceded by [GABA] elevations in the pyramidal cell layer}

Whilst the 'sniffer' patch can detect [GABA] e changes with high sensitivity, it reports tissue-average [GABA] values near the slice surface, potentially far away from the source. To understand whether [GABA] displays similar dynamics within the pyramidal cell layer (one of the main axon target areas of FS interneurons), at a depth inside the tissue, we set out to examine the relationship between interictal discharges and $[G A B A]_{e}$ using the recently developed optical GABA sensor iGABASnFR ${ }^{23}$. 
a

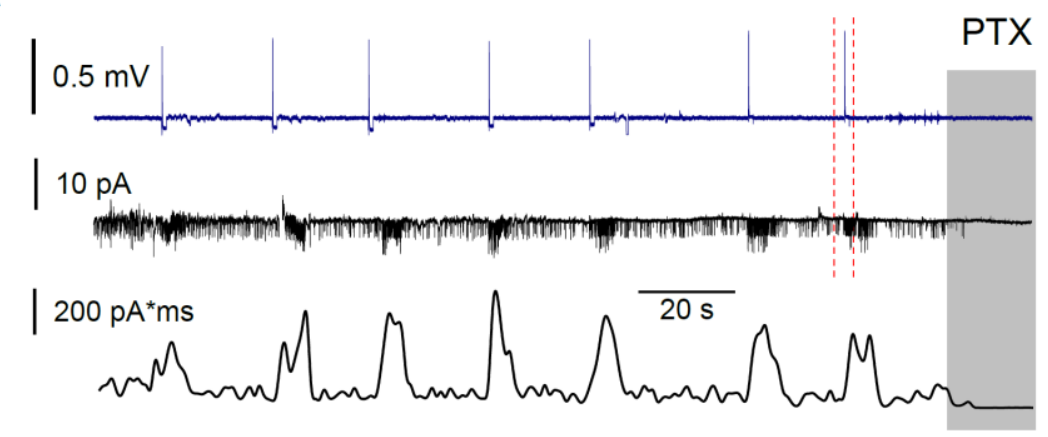

b

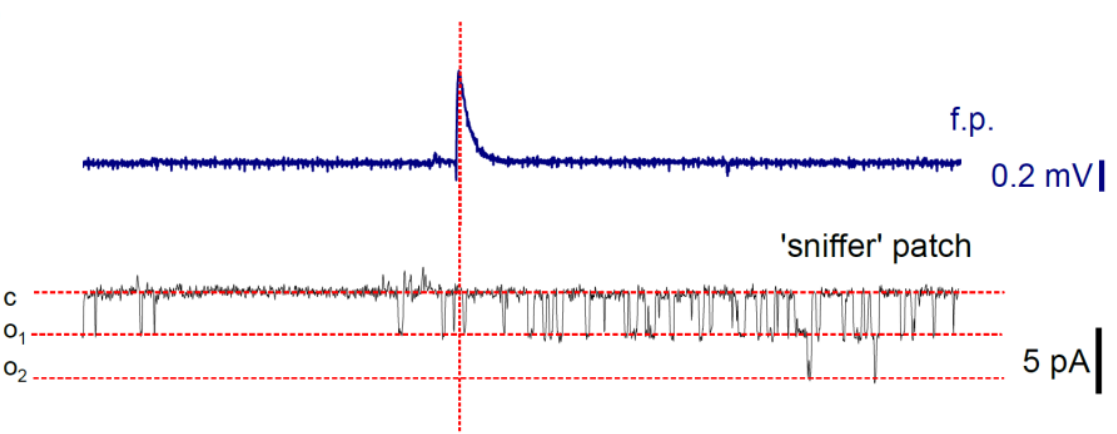

C
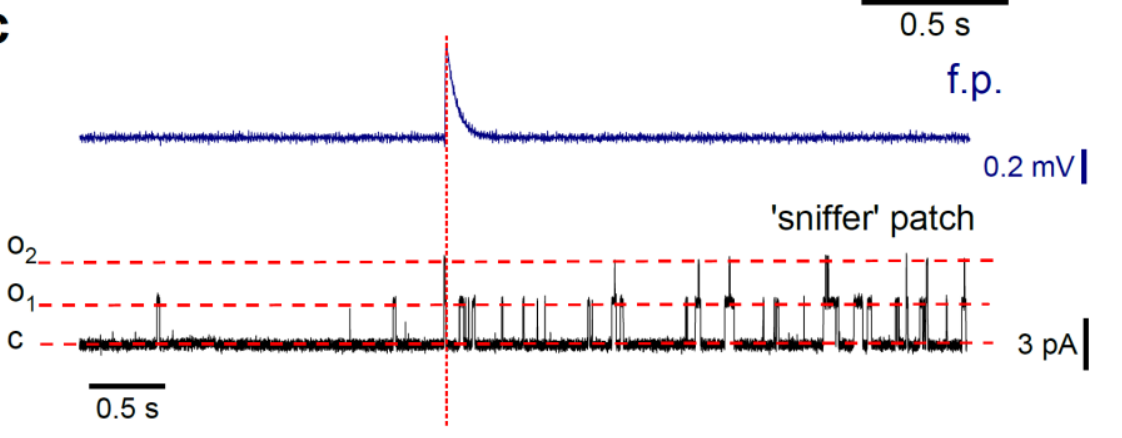

\section{d}

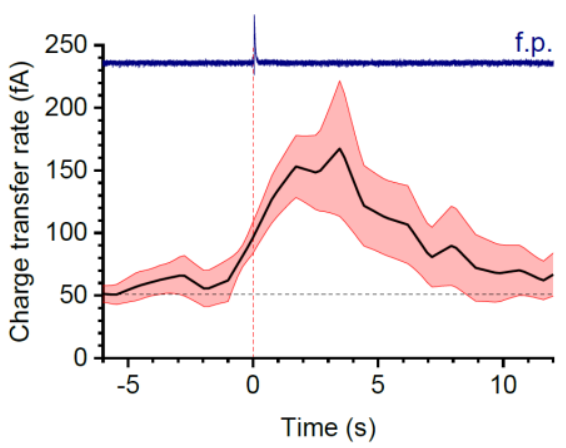

e

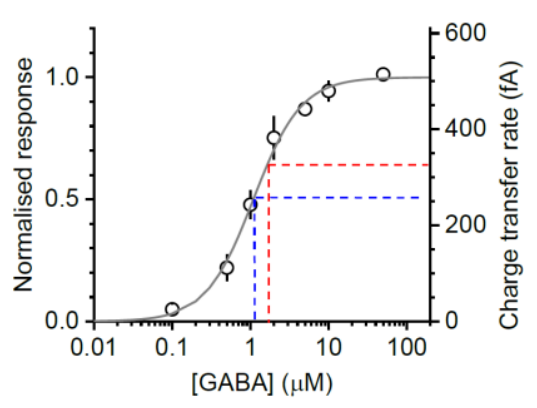

Figure 1. Rhythmic fluctuations of extracellular GABA during interictal activity in the hippocampal area CA1. a, Local field potentials (LFP, top trace), sniffer-patch single-channel activity (middle), and charge dynamics (bottom) during interictal discharges in hippocampal slices; greay shade, picrotoxin (PTX) application at the end of trials. $\mathbf{b}$, Fragment in a (between dotted lines) expanded to show the LFP (top) and patch recording (black) details; high chloride pipette solution was used to record inward $G_{A B A} R$-mediated channel openings $\left(V_{m}=-70\right.$ $\mathrm{mV})$. c, Similar setting to $\mathbf{b}$, but with low chloride solution to document outward currents $\left(\mathrm{V}_{\mathrm{m}}=\right.$ $0 \mathrm{mV}$ ). $\mathbf{d}$, Averaged time course of charge transfer rate (equal to current; mean \pm s.e.m. for slice-average traces, $n=6$ slices) at the onset of individual LFP bursts (vertical dashed line, blue trace). e, Normalised signal and charge transfer values recorded in sniffer patches at different $[G A B A]_{e}$ (mean \pm s.e.m.; $n=4-6$, for individual [GABA] $]_{e}$ values); fit to a sigmoidal curve; dashed lines depict the average baseline (blue) and peak (red) values recorded. 
We therefore expressed the sensor in a proportion of CA1 and CA3 pyramidal cells under the hSynapsin-1 promoter, in organotypic hippocampal slices (Fig. 2a; Methods): sparse expression of iGABASnFR helped to minimise any potential buffering effects on the dynamics of extracellular GABA $29,30$.

We next imaged iGABASnFR fluorescence within microscopic regions of interest (ROIs) near the periphery of individual pyramidal cell somata using two-photon excitation microscopy, as described earlier ${ }^{31}$ while recording field potentials nearby

(Fig. 2a). Periodic interictal events were consistently accompanied by transient rises of fluorescence (Fig. 2b, bottom trace). The analysis of field excitatory post-synaptic potentials (fEPSPs) and fluorescent iGABASnFR signals at higher temporal resolution revealed that the latter consistently increased before interictal spikes. The average interval from the increase in iGABASnFR to the fEPSP spike was $327 \pm 77$ ms (mean \pm s.e.m., $n=7$ slices; iGABASnFR signal onset is taken at $20 \%$ of its peak, for conservative lag estimation; Fig. 2c-e; Extended data Fig. 2). The latency from the increase in $[G A B A]_{e}$ to the fEPSP spike would be orders of magnitude greater than the lag from a hypothetical pacemaker elsewhere in the hippocampal slice, arguing against the hypothesis that iGABASnFR is merely detecting a barrage of feed-forward inhibition.

During these cycles, the iGABASnFR signal peaked at $0.024 \pm 0.002 \Delta F / F$ (mean \pm s.e.m.; Fig. $2 f$, left ordinate). It has earlier been reported that membrane-bound iGABASnFR expressed extracellularly in hippocampal neurons, and calibrated using clamped [GABA] levels, shows an affinity of $\sim 30 \mu \mathrm{M}$, with a saturating signal of $\sim 0.7$ $\Delta F / F^{23}$. In accordance with the Hill equation, and with a basal [GABA] level of $\sim 0.3$ $\mu \mathrm{M}$ (Fig. 1), this suggests that $0.024 \Delta F / F$ signal corresponds to a $\sim 1.2 \mu \mathrm{M}$ rise in $[G A B A]_{e}$, in excellent agreement with the 'sniffer' patch data (Fig. 1d-e). We note that the $[G A B A]_{e}$ transients reported with iGABASnFR are relatively noisy, most likely because they represent microscopic ROls, in contrast to the [GABA] e sensed (and time-filtered) by the 'sniffer' patch over larger tissue volumes.

\section{Biophysical basis of [GABA] e-dependent network rhythms}

To understand the role of $[\mathrm{GABA}]_{\mathrm{e}}$ fluctuations in the generation of periodic interictal discharges, we simulated a network of FS hippocampal interneurons, with a stochastic excitatory input to individual cells (Fig. 3a; Methods), by building upon our previous network models 14, 26, 27 . An important feature of the network was that interneuronal discharges generated both the IPSCs in individual cells and the [GABA] e rises that contributed to Gtonic across the cell population $26,27$. 
a

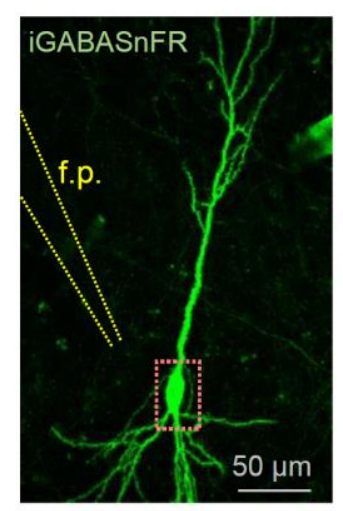

C
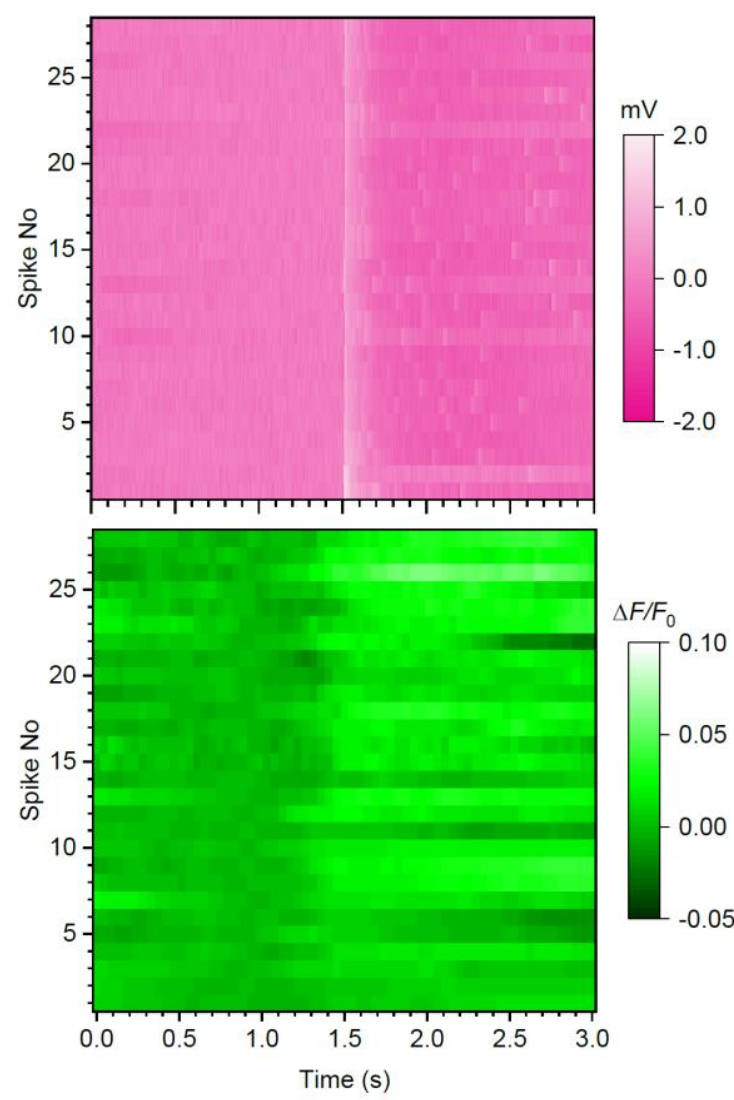

b

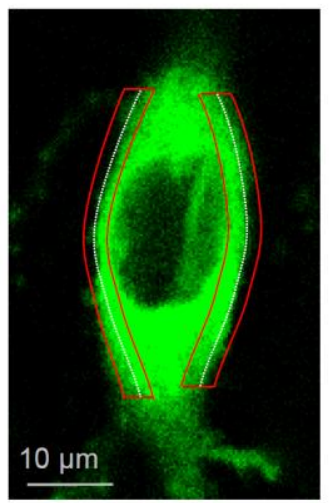

fEPSP

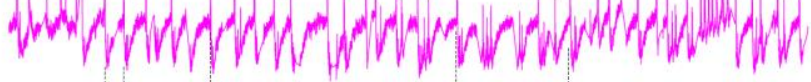

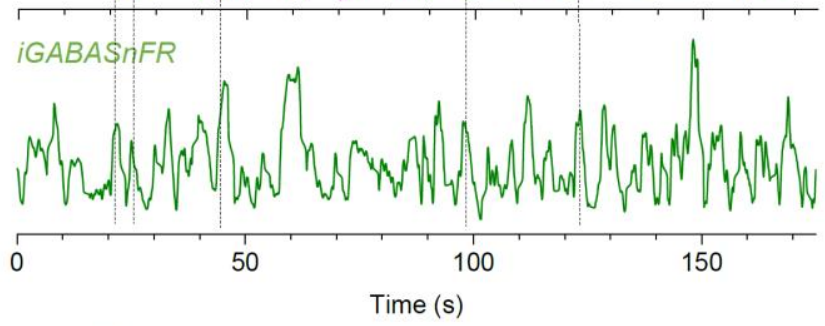

d

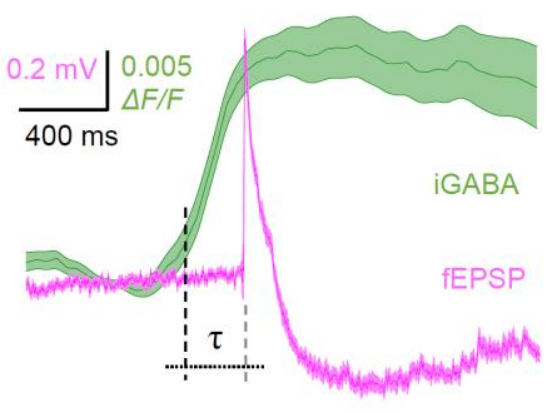

e

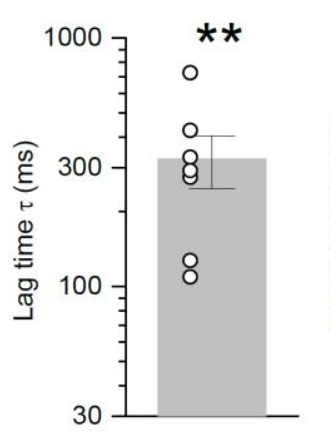

f

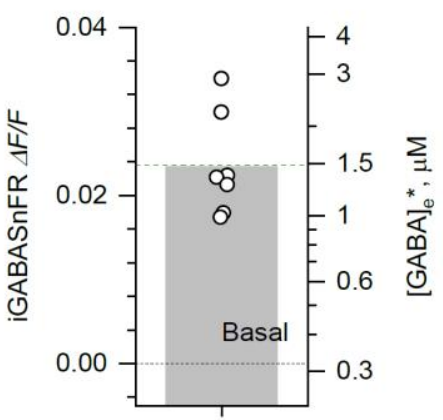

Figure 2. Optical registration of [GABA] $]_{\mathrm{e}}$ waves during interictal events in hippocampal slices. a, A CA1 pyramidal cell expressing iGABASnFR with a field pipette electrode (f.p., left) and two imaging ROls near the cell soma periphery (right, zoomed-in fragment shown by red rectangle on the left; $\lambda_{x}{ }^{2 p}=910 \mathrm{~nm}$ ). $\mathbf{b}$, One-slice example, fEPSPs (top) and iGABASnFR signal (bottom) recorded during interictal spikes. c, Diagram showing 28 interictal fEPSPs aligned with respect to their peak times (top), and the corresponding iGABASnFR signals (bottom; false colour scale; one-slice example). d, Summary of recordings shown in c, at expanded time scale: traces, mean \pm s.e.m. $(n=28)$; dotted lines, lag time $\tau$ between the $20 \%$ peak rise time of iGABASnFR signal and peak time of fEPSP, as indicated. e, Summary for $\tau$ values obtained in seven slices (mean \pm s.e.m; dots, data from individual slices; ${ }^{*} p=0.0052$, paired Student's $t$-test). f, Average peak iGABASnFR signal $\Delta F / F$ values for seven experiments as in e (left ordinate; column, mean), and the corresponding estimated [GABA] values $\left([G A B A]_{e}{ }^{*}\right.$, right ordinate). 
$G_{\text {tonic }}$ was thus set as a monotonic function of [GABA] (Methods), which in turn was determined by (i) a scalable [GABA] increment upon each individual synaptic discharge, and (ii) GABA removal by the main GABA transporter GAT-1 yielding an exponential [GABA] e decay ${ }^{5,32}$ (Methods).

We simulated networks of varied sizes and properties, including a plausible range of GABA release and uptake rates. First, simulations revealed that under constant Gtonic, cells fire stochastically, at a near-constant average frequency, showing little synchronization (Fig. 3b). In striking contrast, when [GABA]e (hence Gtonic) dynamics were driven by interneuronal spiking, the network consistently exhibited spontaneous periodic activity patterns, over a wide range of frequencies depending on several key parameters: the excitatory drive, synaptic connectivity strength, and the GABA release rate (Fig. 3c-d; Extended Data Fig. 3). Simulations showed a robust, predictable relationship between interneuronal spiking, [GABA] , and $G_{\text {tonic }}$ during periodic busts of activity (Extended Data Fig. 4a-d). Interestingly, larger networks (containing 400-1500 cells) tended to have a more regular activity cycle (Extended Data Fig. 4), which is consistent with classical theory predicting less variability near a system's Hopf bifurcation point ${ }^{33}$, which occurs towards the peak of Gtonic.

Finally, we explored the relationship between cell spiking activity, Gtonic, and [GABA] in a twinned network configuration that incorporated both interneurons and principal cells (PCs) equipped with $\mathrm{GABA}_{A} \mathrm{Rs}$. An initial exploration of connectivity parameters has indicated that such networks show stable behaviour under external stimuli when synaptic connectivity between the two networks (average synaptic conductance $G_{i e}$, interneurons to PCs, and $G_{\mathrm{ei}}$, PCs to interneurons) were, on average, two orders of magnitude lower than intra-network connectivity ( $G_{\text {ee }}$ and $\left.G_{i i}\right)$. Both interneuronal and PC networks were kept activated by stochastic excitatory conductance, $g_{\mathrm{si}}$ and $g_{s e}$, respectively. We found that, over a range of $g_{s e}$ values, behaviour of the PC network was distinctly sensitive to [GABA] e-dependent Gtonic generated by the interneuronal network. PCs showed brief, highly-synchronised bursts of activity that followed periodic rises of interneuronal spiking (Fig. 3e). Strikingly, the PC spiking bursts occurred on the ascending phase of [GABA] waves (Fig. 3f), thus displaying some distinct features of the epileptiform events that we observed experimentally (Fig. 1d, Fig. 2d).

\section{Self-maintained waves of tonic GABAA conductance in hippocampal slices}

To examine the validity of causal relationships revealed by the modelling, we carried out several experiments. First, we asked whether the dynamics of Gtonic predicted by simulations (Fig. 3a-d) occur in hippocampal circuits in the absence of glutamatergic transmission. We therefore recorded $\mathrm{GABA}_{A} \mathrm{R}$-mediated currents from CA1 pyramidal neurons (whole-cell; unless specified otherwise, these and subsequent acute slice experiments were carried out in the presence of $50 \mu \mathrm{M}$ NBQX, $10 \mu \mathrm{M} A P V$, and $1 \mu \mathrm{M}$ CGP52432, to block AMPA, NMDAR, and GABAв receptors, respectively). 


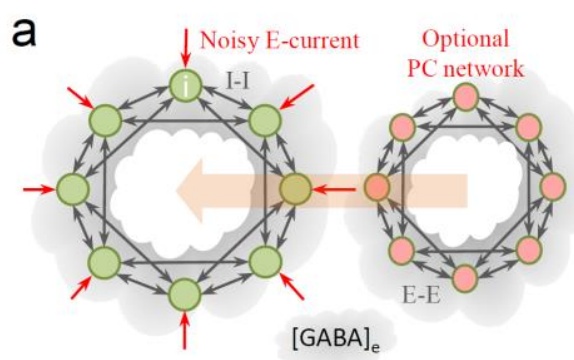

C

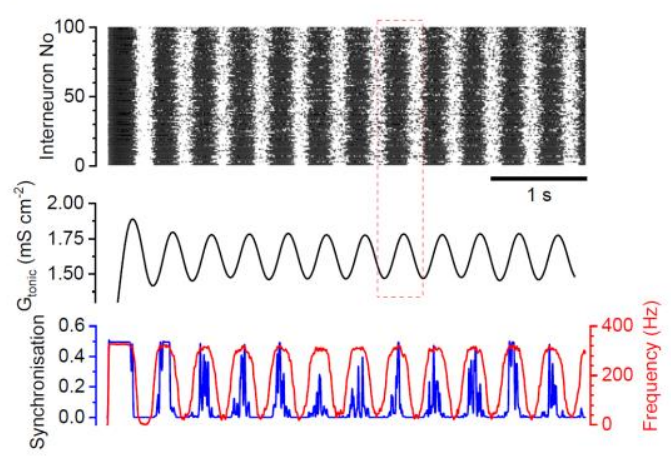

e

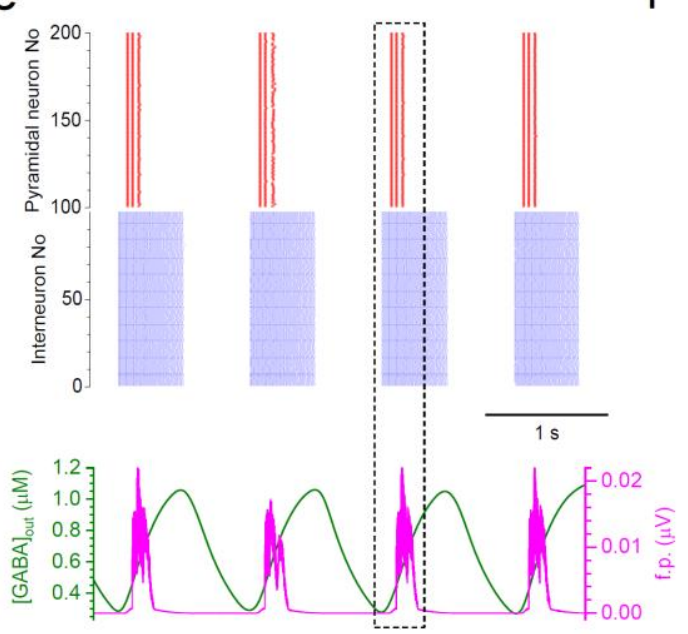

b

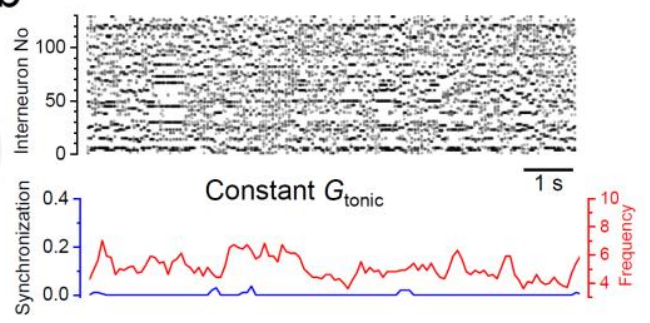

d

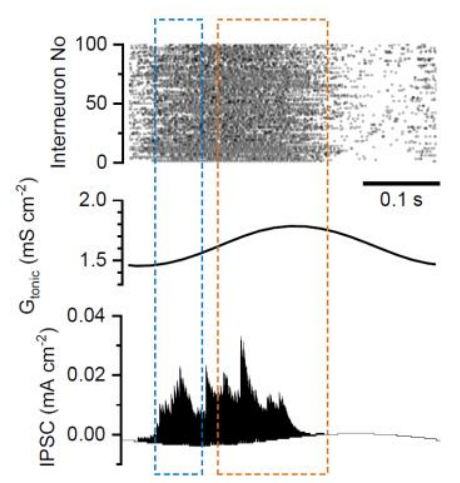

f
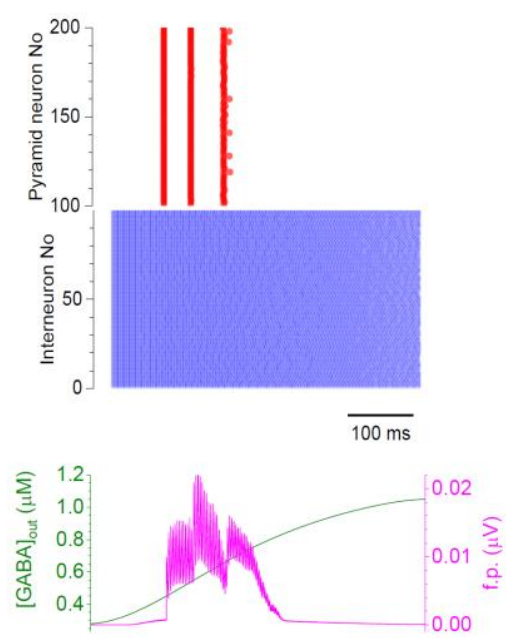

Figure 3. [GABA] dependent $G_{\text {tonic }}$ robustly drives rhythmic activity of a modelled interneuron network across a range of parameters. a, Schematic, FS interneuron network model (I-I, inhibitory synaptic connections), with external excitatory inputs (E-current), volume-transmitted [GABA] signal generating $G_{\text {tonic }}$, and an optional network of pyramidal cells incorporated (E-E, excitatory connections). b, Raster plot of interneuronal network spiking (top), network synchronisation coefficient (bottom, blue), and time course of average spiking frequency (bottom, red) for simulated network with constant $G_{\text {tonic }}$ value $\left(0.44 \mathrm{mS} \mathrm{cm}^{-2}\right)$. c, Raster plot as in b but with $G_{\text {tonic }}$ driven by $[G A B A]_{e}$ (middle) calculated from

integrated interneuronal discharges; network synchrony and mean network frequency show clear periodicity (bottom). Key model parameters: cell number $N=100$, intra-network peak synaptic conductance $G_{i i}=0.1 \mathrm{nS} \mathrm{cm}^{-2}$; E-currents (Poisson series) with average synaptic conductance $g_{\mathrm{s}}=0.02 \mathrm{mS} \mathrm{cm}^{-2}$, decay constant tau $=3 \mathrm{~ms}$, and frequency $f_{s}=100 \mathrm{~Hz} ; G_{\text {tonic }}$ reverse potential $V_{G A B A}=-56 \mathrm{mV}$, GABA release factor $A_{f}=1.35 \times 10^{-7} \mathrm{nS} \mathrm{cm}^{-2} \mathrm{~ms}^{-1}, G_{\text {pump }}$ $=0.003 \mathrm{~s}^{-1}$ (see Methods for further detail). d, Fragment from $\mathbf{c}$ (red dotted rectangle) enlarged, with the integrated IPSC time course (bottom). Blue and orange dotted rectangles indicate high-frequency non-synchronised, and oscillating and synchronised IPSC periods, respectively. e, Top: Raster plot for a twinned network (red, pyramidal neurons; blue, interneurons), with weak internetwork (I-E and I-E) and strong intra-network (I-I and E-E) connections. Bottom: Time course of field potential (f.p.; simulated for pyramidal neurons at $250 \mu \mathrm{m}$ from the network, tissue conductance of $100 \mathrm{mS} \mathrm{cm}^{-1}$; Methods) and [GABA] $]_{\mathrm{e}}$, as indicated. Key model parameters: $N=200$ (100 pyramidal cells and 100 interneurons), $G_{i i}=$ $0.216 \mathrm{mS} \mathrm{cm}^{-2}, G_{\mathrm{ee}}=0.003 \mathrm{mS} \mathrm{cm}^{-2}$; Inter-network peak synaptic conductance $G_{\mathrm{e}}=0.00012$ $\mathrm{mS} \mathrm{cm} \mathrm{cm}^{-2}$ and $G_{\mathrm{ie}}=0.00064 \mathrm{mS} \mathrm{cm}^{-2}$, E-currents (Poisson series) $g_{\mathrm{si}}=0.3 \mathrm{mS} \mathrm{cm}^{-2}$, tau $=3 \mathrm{~ms}$, $f_{s}=20 \mathrm{~Hz}$; l-current (Poisson series) $g_{\mathrm{se}}=0.003 \mathrm{mS} \mathrm{cm}^{-2}$, decay constant tau $=3 \mathrm{~ms}, f_{s}=20 \mathrm{~Hz}$, $V_{\text {GABA }}=-58 \mathrm{mV}, A_{f}=2 \times 10^{-7} \mathrm{nS} \mathrm{cm} \mathrm{ms}^{-1}, \mathrm{G}_{\text {pump }}=0.003 \mathrm{~s}^{-1}$. f, Fragment of network activity shown in e (dashed rectangle) expanded, notations as in $\mathbf{e}$. 
Elevating extracellular $\left[\mathrm{K}^{+}\right]$to $10 \mathrm{mM}$ in nominally $\mathrm{Mg}^{2+}$-free medium, to increase interneuronal activity, elicited outward shifts in the holding current, which were sensitive to picrotoxin (a non-competitive GABA $A_{A}$ channel blocker; Fig. 4a, b), and consisted of brief transients riding on periodic low frequency waves (Fig. 4c). The frequency of the slow $\mathrm{GABA}_{A} \mathrm{R}$-mediated waves $(0.2-0.3 \mathrm{~Hz})$ was similar to the periodic [GABA] e transients observed with GABA imaging (Fig. 2b). Consistent with the network model (Fig. 3a-d, Extended Data Figs. 3-4), the brief GABAAR-mediated currents, which most likely reflect individual interneuron discharges, tended to increase in intensity before the peak of the slow waves reflecting Gtonic (hence [GABA]e) (Fig. $4 c-e)$.

\section{Synchronisation of fast-spiking PV+ interneurons during GABA waves}

To understand better the dynamics of synchronised interneuronal activity during GABA waves, we simultaneously monitored spiking activity in two FS PV+ interneurons (in cell-attached mode) and GABA $A$-mediated currents in a CA1 pyramidal cell (wholecell mode), with ionotropic glutamate receptors blocked (Fig. 5a). Clear rhythmicity of interneuron activity was documented in three out of nine experiments (wave-like activity occurred in both patched PV interneurons, i.e., 6 out of 18 recorded interneurons), suggesting that a sizeable proportion of hippocampal cells contribute to the network waves reported above. In such experiments, quasi-periodic bursts of interneuron spiking (Fig. 5b; magenta) were observed together with the corresponding fluctuations of GABAergic currents in the pyramidal neuron (Fig. 5b, green). When the degree of synchronization among interneurons was quantified, there was a positive correlation between the spiking frequencies of the two PV+ cells and the synchronization parameter (example in Fig. 5c). Overall, experiments that featured prominent rhythmic waves also showed a significant interneuron synchronizationspiking correlation, whereas experiments that did not exhibit rhythmicity showed no such correlation (summary in Fig. $5 d$; examples of experiments without rhythmicity and related data in Fig. 5e-f).

\section{Spiking of principal neurons is synchronised shortly after the peak of interneuronal firing}

Next, to examine how interneuronal network activity modulates firing of principal cells, we recorded one CA1 or CA3 pyramidal cell in cell-attached mode while voltageclamping another pyramidal neuron nearby to monitor its GABAergic input (at $10 \mathrm{mM}$ $\left.\mathrm{K}^{+}\right)$. Pyramidal cell spiking showed a characteristic lull during the rising phase of the $G_{\text {tonic }}$ wave before rebounding as the latter reached a peak (Fig. 6a left). Blocking $\mathrm{GABA}_{A} \mathrm{Rs}$ with picrotoxin abolished this periodic fluctuation in spike frequency (Fig. $6 \mathrm{a}$ right). 
a
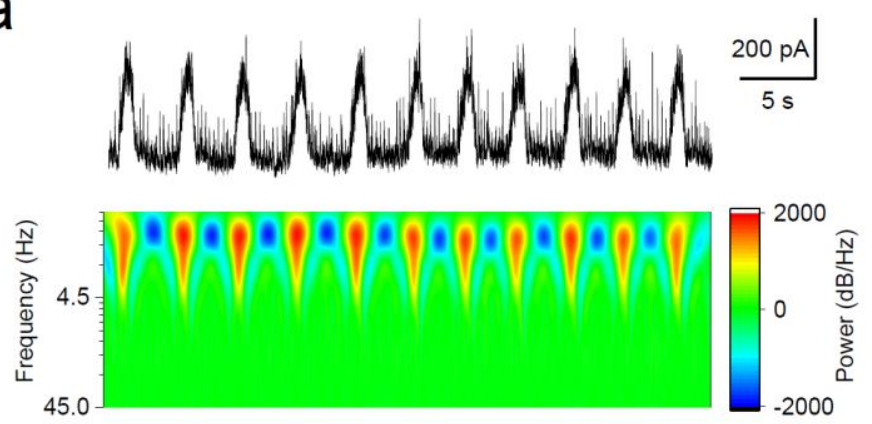

b

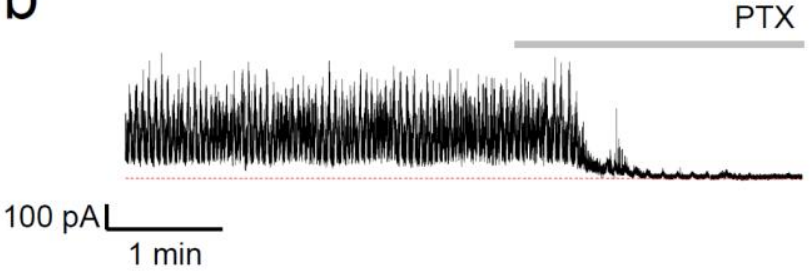

C
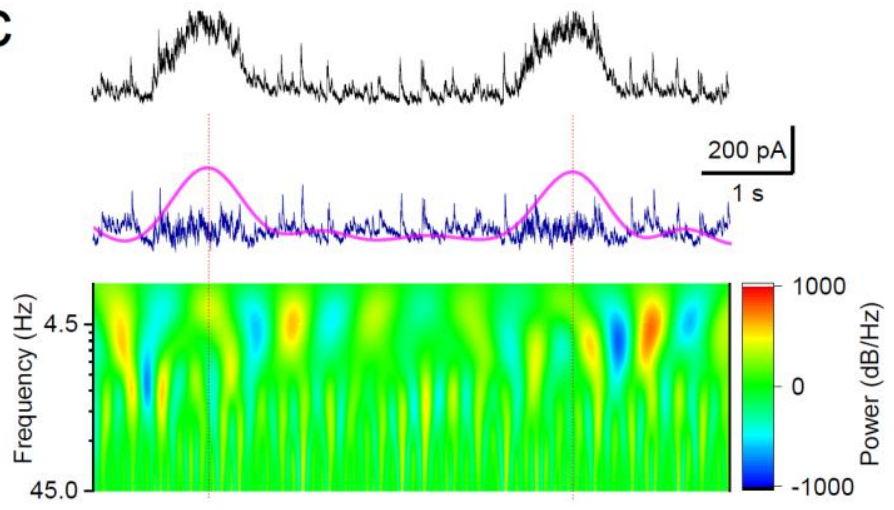

d

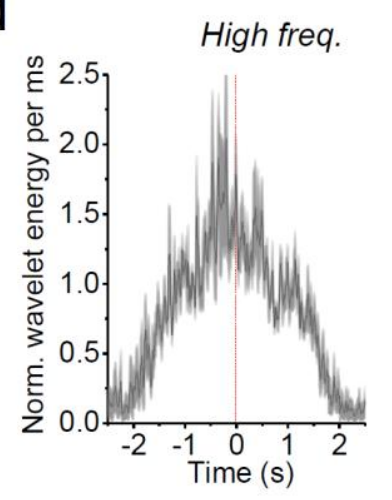

e

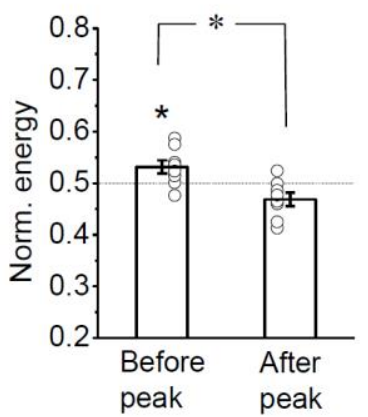

Low freq.
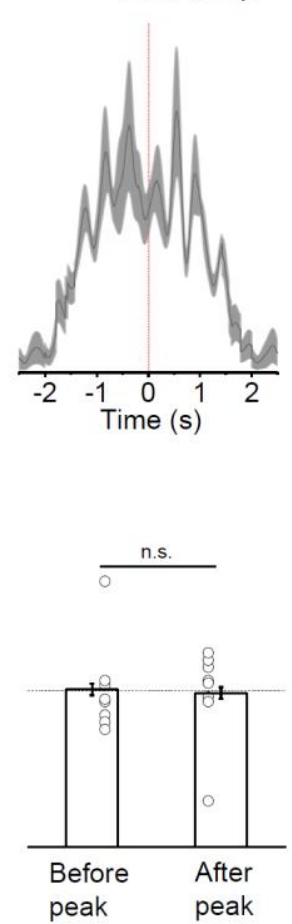

Figure 4. Experimentally induced slow wave-like oscillations of interneuronal activity. a, Trace, example of $G A B A_{A} R$-mediated currents received by a CA 1 pyramidal neuron and the corresponding spectrogram (bottom; voltage-clamp with low intracellular $\mathrm{Cl}^{-} ; \mathrm{V}_{\text {hold }}=0 \mathrm{mV}$ ). $\mathbf{b}$, Slow wave-like oscillations as in a are readily blocked by the $G A B A_{A} R$ antagonist picrotoxin (PTX). c, Traces as in a expanded: raw data (black) and its $2 \mathrm{~Hz}$ low-pass (i.e., $G_{\text {tonic }}$; magenta), high-pass (blue) filtered components, and the corresponding spectrogram (bottom). d, Examples of wavelet energy plots showing a consistent increase in the high-frequency component before the peak of $G_{\text {tonic. }}$ e, Statistical summary: bar graphs, signal spectral energy (mean \pm s.e.m, $\mathrm{n}=7$ slices; dots, individual experiments), before and after the $G_{\text {tonic }}$ peak; ${ }^{\star} p<0.05$, paired Student's $t$-test. 
a

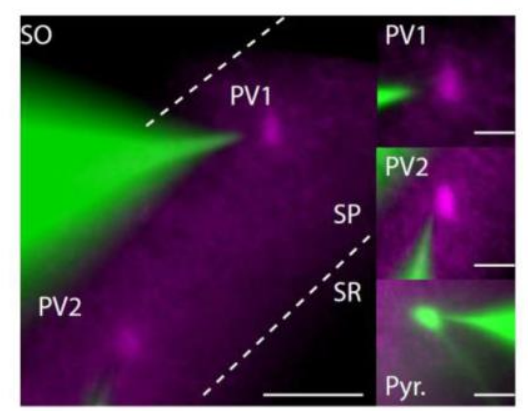

b
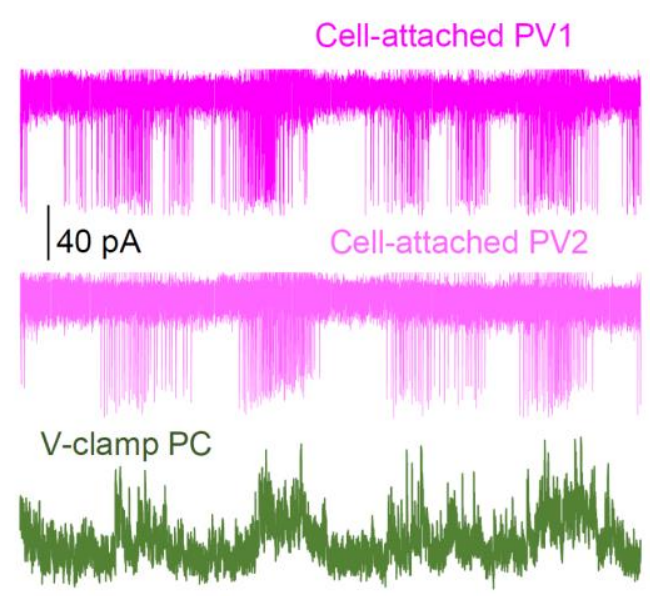

C

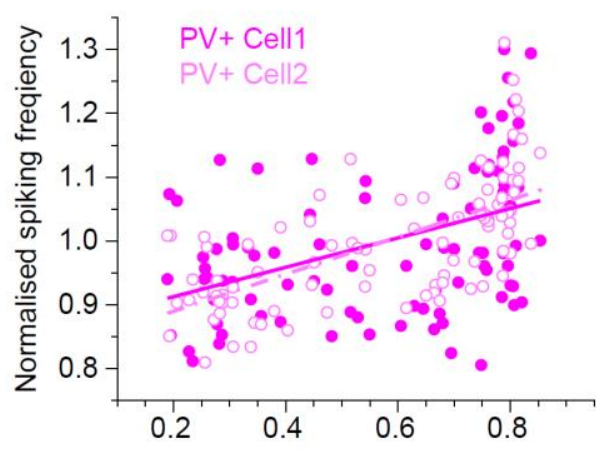

Synchronisation between Cell1 and Cell2

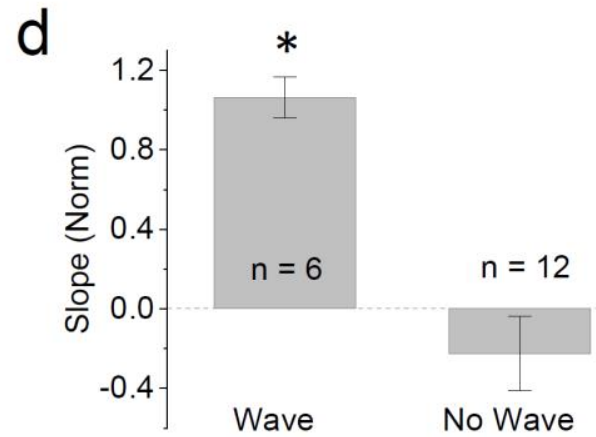

e
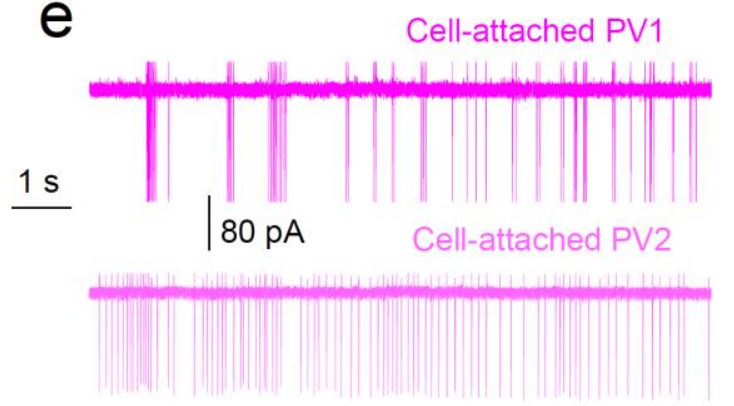

$150 \mathrm{pA}$

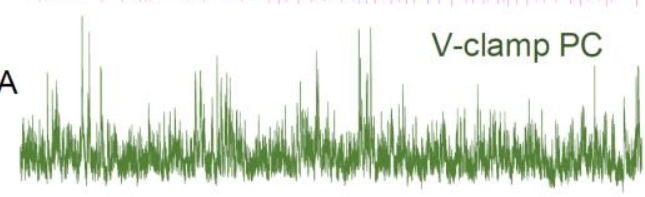

f

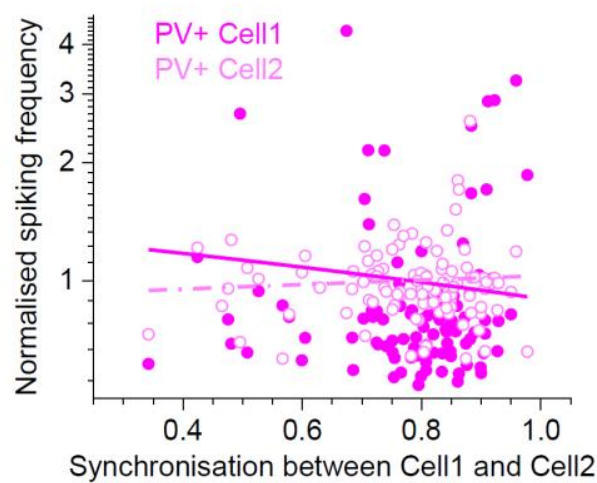

Synchronisation between Cell1 and Cell2

Figure 5. Synchronization of FS PV+ interneurons spiking activity during GABA waves. a, Illustration of dual cell-attached recordings of PV+ interneurons (green: Alexa Fluor 48850 $\mu \mathrm{M}$; magenta: tdTomato) and voltage-clamp recording of a CA1 pyramidal cell (AF488, $50 \mu \mathrm{M})$. Scale bars, $50 \mu \mathrm{m}$ (left) and $20 \mu \mathrm{m}$ (right). b, Example, time course of spiking activity of two simultaneously recorded PV+ cells (top and middle traces), and IPSCs in a pyramidal neuron (bottom, $\mathrm{V}_{\text {hold }}=+10 \mathrm{mV}$ ) in high $\mathrm{K}^{+}$solution $(10 \mathrm{mM})$ and $0 \mathrm{Mg}^{2+}$; rhythmic network waves are present. c, Spiking frequencies for the two PV+ cells (as shown in $\mathbf{b}$ ), against their synchronization coefficient; straight lines, the corresponding linear regressions. d, Summary, regression slope (mean \pm s.e.m.) between $\mathrm{PV}+$ spiking intensity and synchronization parameter in dual-patch recordings, as shown in c; samples with rhythmic waves $(n=6$ cells in 3 slices) and without ( $n=12$ cells in 6 slices) are shown; ${ }^{*} p<0.03$, unpaired Student's ttest. e-f, Examples as in $\mathbf{b}-\mathbf{c}$ with no detectable rhythmic waves; notations as in $\mathbf{b}$-c. 
Thus, a rise in interneuron activity could paradoxically initiate interictal events in pyramidal cells, by synchronising their firing, which was consistent with the sequence of events reported earlier in the cortical circuitry ${ }^{20}$. To test this further, we depolarised, synchronously and en masse, $\mathrm{PV}+$ interneurons in the CA1 area using wide-field optogenetic stimulation (Fig. 6b). First, we confirmed that $\mathrm{PV}+$ interneurons expressing channelrhodopsin-2 (ChR2-H134R-eYFP) reliably responded to $1 \mathrm{~ms}$ light pulses (Fig. $6 c$ ). We next asked whether optogenetic stimulation of $P V+$ cells could induce interictal events in the hippocampal slice superfused with $5 \mathrm{mM}\left[\mathrm{K}^{+}\right]$and nominally $\mathrm{Mg}^{2+}$-free solution. Indeed, a single $1 \mathrm{~ms}$ light pulse evoked large GABAAR IPSCs in CA1 pyramidal neurons, with coincident epileptiform discharges (field potentials) which were indistinguishable from spontaneous interictal events (Fig. 6d, Extended Data Fig. 5a). Again, this observation is consistent with that seen under similar circumstances, albeit a different experimental model of interictal activity, in cortical slices ${ }^{21}$.

Blocking $\mathrm{GABA}_{\mathrm{A}}$ receptors by bath application of picrotoxin completely abolished light evoked interictal spikes, confirming a requirement for GABA release from $\mathrm{PV}+$ cells (Extended Data Fig. 5a). In addition, optogenetic stimulation applied at a frequency between 0.03 and $0.2 \mathrm{~Hz}$ consistently evoked such events with $\sim 0.8$ probability whereas above $0.2 \mathrm{~Hz}$ (close to natural periodicity, $0.14 \pm 0.03 \mathrm{~Hz}$ ) this induction became less reliable, decreasing to a probability of $\sim 0.1$ at $5-10 \mathrm{~Hz}$ (Fig. 6e). Finally, light stimuli also evoked bursts of action potentials in pyramidal neurons, with a delay similar to that of interictal events ( $42 \pm 3 \mathrm{~ms}$ vs. $43 \pm 5 \mathrm{~ms}$; Extended Data Fig. 5b). Thus, an abrupt release of PV+ cell inhibition (caused by light termination) triggered synchronous pyramidal neuron discharges.

\section{Evoked [GABA] waves can trigger network rhythms}

We next asked whether an externally enforced wave of GABA release could entrain interictal discharges. We therefore carried out progressive excitation of $\mathrm{PV}+$ cells using optogenetic stimulation ramps (1-2 s duration, comparable to spontaneous GABA waves) while recording IPSC activity in CA1 pyramidal cells (Fig. 7a). A high-resolution analysis revealed that progressive optogenetic stimulation of PV+ cells elevated IPSC activity only to a certain point, after which the IPSC frequency rapidly dropped (Fig. 7b). Similar events could be seen leading to an inter-ictal spike (Fig. 7c-d). Indeed, in five out of eight experiments, we clearly observed wave-like GABAAR-mediated currents induced by optogenetic ramp stimulation, with a probability of inducing interictal spikes of $0.25 \pm 0.09$ with a delay of $831 \pm 47 \mathrm{~ms}$ (detection window between 500 and 100 ms post-stimulation). In contrast, the three experiments without lightevoked GABA waves exhibited a much lower likelihood of triggering epileptiform discharges $(0.06 \pm 0.04)$. 
a

PC cell-attached

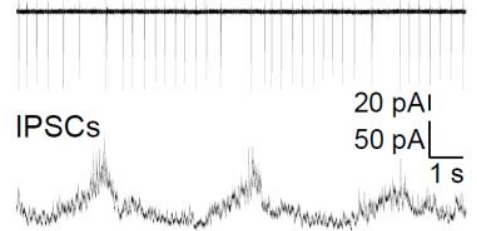

b

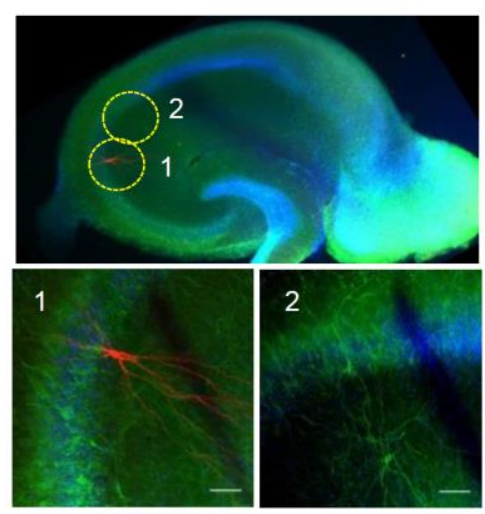

d
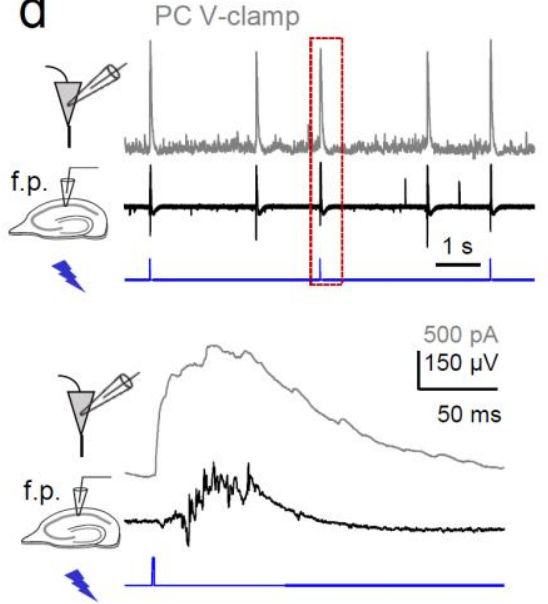

in PTX

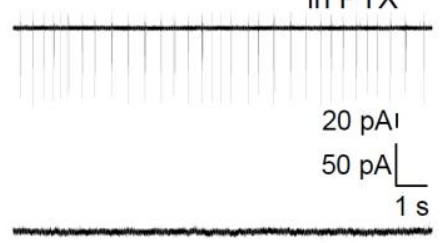

C
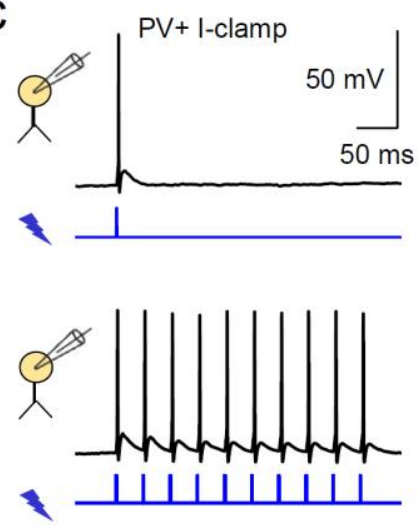

e

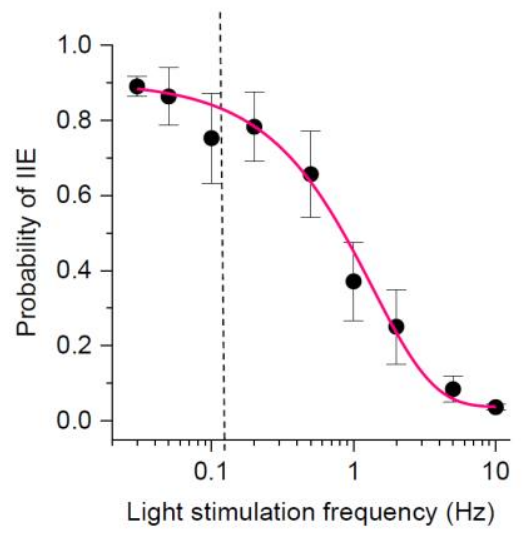

Figure 6. Short photoactivation of PV+ interneurons evokes epileptiform burst discharges. a, Simultaneous cell-attached and whole-cell recordings of action potentials (top trace) and GABA $A_{A}$ R-mediated IPSCs (bottom) during [GABA] waves (left) and after application of picrotoxin (PTX). b, Transverse hippocampal slice of PV:: Cre X Ai32 mouse(top): green fluorescence shows ChR2 expression in PV+ cells against DAPI (blue) nuclear counterstain (Methods). Images 1-2 (dotted circles above): post hoc identification of a CA3 pyramidal neuron (biocytin fill, red; magnified in 1) after a whole-cell recording shown below in d; scale bars: $20 \mu \mathrm{m}$. c, Current-clamp recordings from a PV+ interneuron activated by a single (left) or repetitive (right) blue light pulses (1 ms, $470 \mathrm{~nm}$ ). d, Example of pyramidal neuron IPSCs (grey trace, top; $\mathrm{V}_{\text {hold }}=+10 \mathrm{mV}$ ) and field potentials (black trace, middle): photo-stimulation of $\mathrm{PV}+$ cells (blue trace, bottom; $1 \mathrm{~ms}$ pulse) triggers large $\mathrm{GABA}_{A} \mathrm{R}$ currents and epileptiform bursts similar to those occurring spontaneously (e.g., Fig. 1); $5 \mathrm{mM} \mathrm{K}+, 0 \mathrm{mM}$ $\mathrm{Mg}^{2+}$ solution. e, Statistical summary: Probability of evoking an inter-ictal event (IIE, mean \pm s.e.m., $n=3-7$ slices for individual probability values, mean delay between the pulse and IIE: $43 \pm 5 \mathrm{~ms}, 1 \mathrm{~ms}$ pulses) as a function of light stimulation frequency; the average frequency of spontaneous IIE $(0.14 \pm 0.03 \mathrm{~Hz}, \mathrm{n}=7$ slices, dotted line) falls within the range of optimal frequencies to entrain the network with light stimuli; at $>0.2 \mathrm{~Hz}$ stimulation, the network enters into a refractory state. 
To understand the biophysical basis of this phenomenon, we asked whether in our simulated network the Gtonic dynamics on its own could drive network synchronization and rhythmicity. We therefore forced Gtonic to follow a $1 \mathrm{~Hz}$ sine wave, and found that cell firing indeed peaked at Gtonic troughs, which were in turn preceded by maximal firing synchronicity (Extended Data Fig. 6), in line with experimental data. Thus, increased interneuronal activity initially boosts network inhibition, followed by prevailing disinhibition (likely due to shunting) - at which point synchronised network discharges are likely to occur, as generally predicted by our theoretical model.

\section{GABA uptake controls the frequency of periodic epileptiform events}

The overall rate of GABA uptake determines how rapidly [GABA] returns to the equilibrated basal level after any possible [GABA] rises. At the same time, changes in the uptake rate should have little effect on the rapid activation of synaptic receptors ${ }^{34}$. Therefore, if the speed of GABA uptake affects network activity, the underlying mechanisms must involve changes in [GABA] hence Gtonic. To test this theoretically, we explored a network of 1500 cells (as in Extended Data Fig. 4e-f) and found that the rate of exponential [GABA] decay of $\sim 16 \mathrm{~s}^{-1}$ can drastically reduce the [GABA] dynamic range, thus effectively suppressing activity rhythms (Fig. 8a-b, top panels). Slowing down [GABA] e decay allowed [GABA] to fluctuate rhythmically, with lower frequencies characteristic for lower decay (uptake) rates (Fig. 8a-b).

To assess whether this causality is indeed present in real networks, we monitored interictal events in slices while adding non-saturating concentrations of NO-711, a specific blocker of the main GABA transporter GAT-1. This protocol was to block the progressively increasing fractions of GAT-1, thus slowing down [GABA] reequilibration without affecting phasic GABAergic transmission or the basal (equilibrated ambient) [GABA] level. Strikingly, slowing down [GABA]e uptake / reequilibration reduced the frequency of interictal discharges, in exact correspondence with the modelling predictions (Fig. 8c-d). These results provide compelling evidence that $[\mathrm{GABA}]_{\mathrm{e}}$ waves play a key role in the generation and pacing of network rhythms in interneuronal networks.

\section{DISCUSSION}

We have found that, in the absence of fast glutamatergic signalling, interneuronal networks exhibit rhythmic synchronization at frequencies similar to interictal bursts. We propose that this population behaviour relies on periodic fluctuations of $G$ tonic driven mainly by activity-dependent dynamics of [GABA] e. Inhibitory restraint has earlier been proposed to "break down" due to depolarising block and reversal of the chloride potential. The present study identifies [GABA] fluctuations as an important mechanism underpinning initiation and propagation of ictal activity. 
a

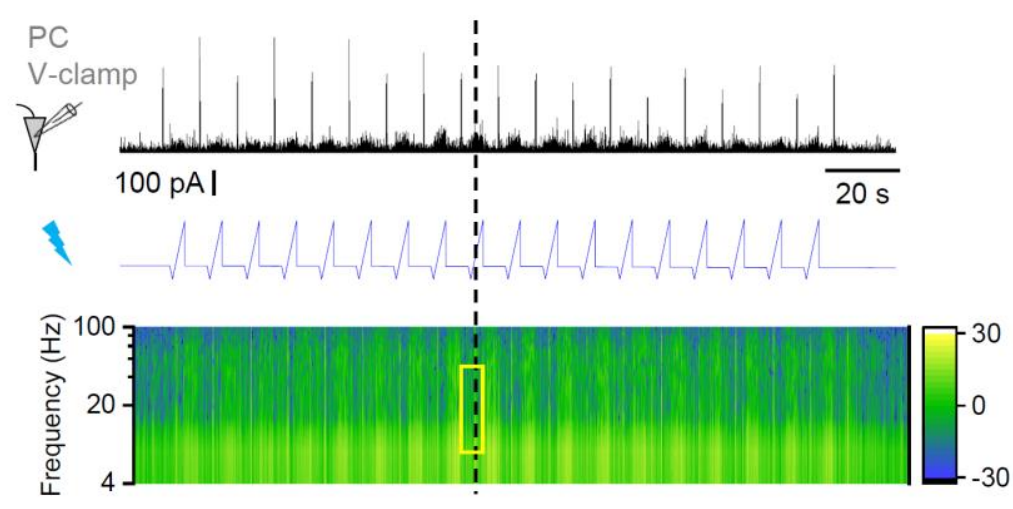

C

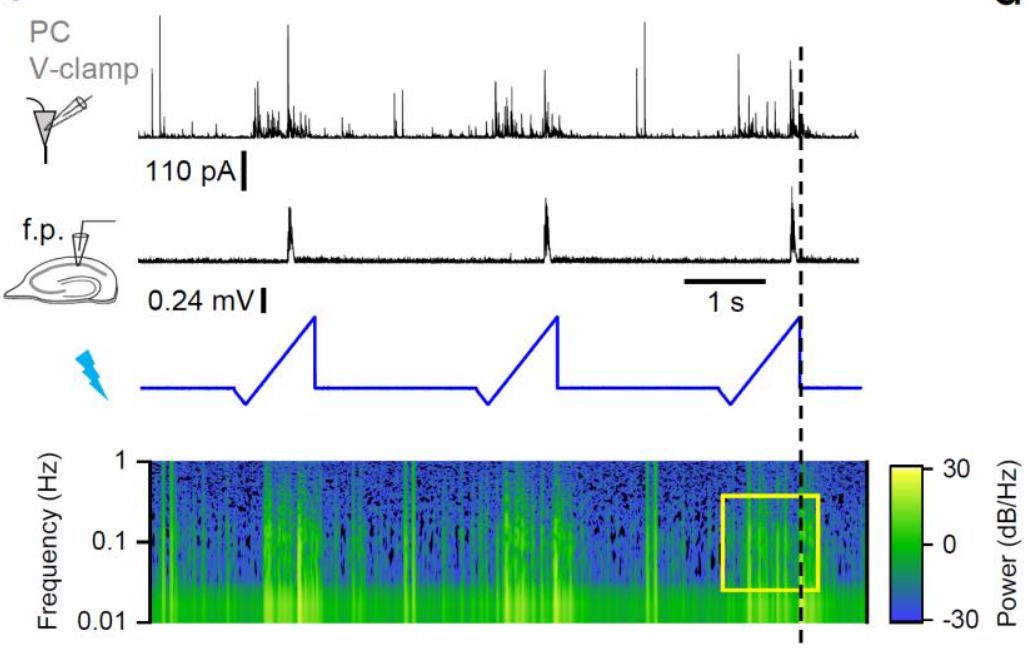

b

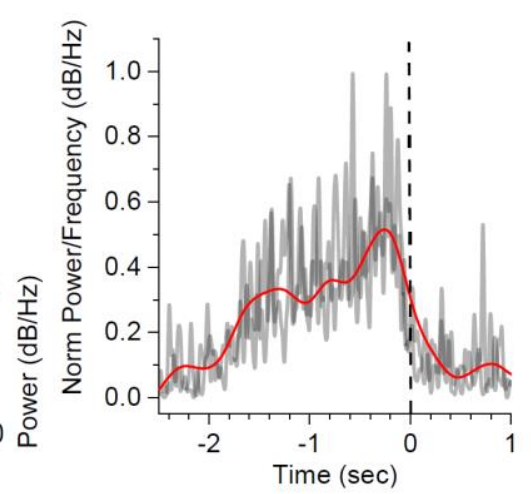

d

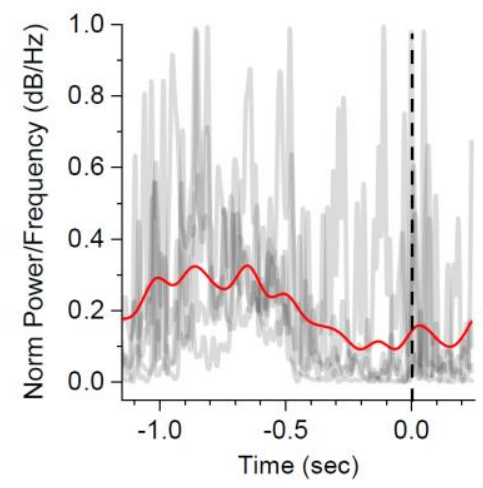

Figure 7. Optogenetically evoked GABA waves induce epileptiform burst discharges. a, $\mathrm{GABA}_{\mathrm{A}} \mathrm{R}$ current in pyramidal neurons ( $\mathrm{V}_{\text {hold }}=+10 \mathrm{mV}$, normal aCSF solution; black trace, top) in response to progressive opto-activation of PV+ interneurons (blue, middle), with the IPSC power spectrum density (bottom). b, Average spectral power density $(20 \times 50 \mathrm{~Hz}$ integration, $\mathrm{n}=18$ ramp stimulations), time window as exemplified by yellow rectangle in a near light intensity peaks (dotted line). c, GABA $\mathrm{A}_{\mathrm{A}}$ current in pyramidal neurons (black trace, top) and local field potentials (middle) during opto-activation (blue trace), with power spectrum density (bottom); note that interictal bursts occur towards the end of the stimulation ramp. d, Average of spectral power density for time window exemplified by yellow rectangle in $\mathbf{c}$; notations as in b. 
a
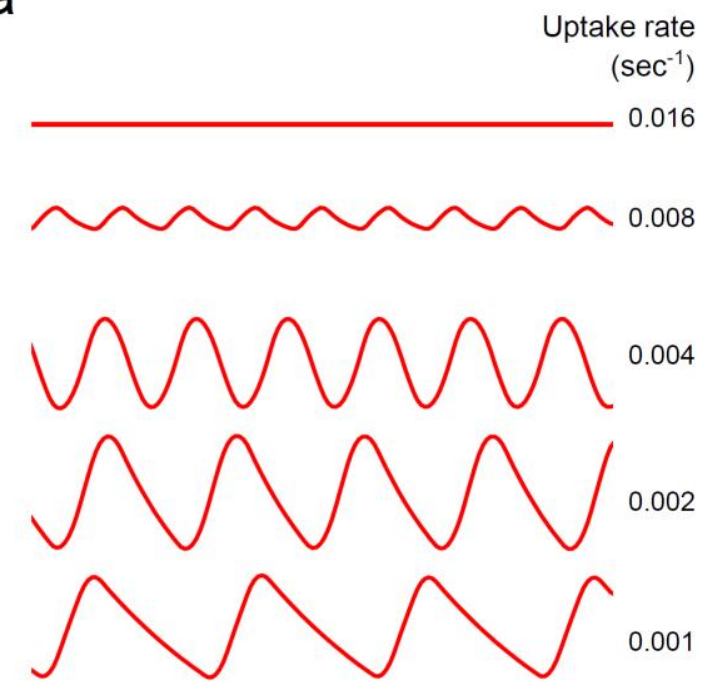

0.001

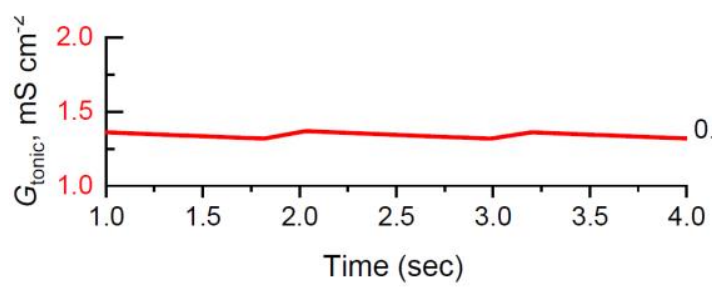

C
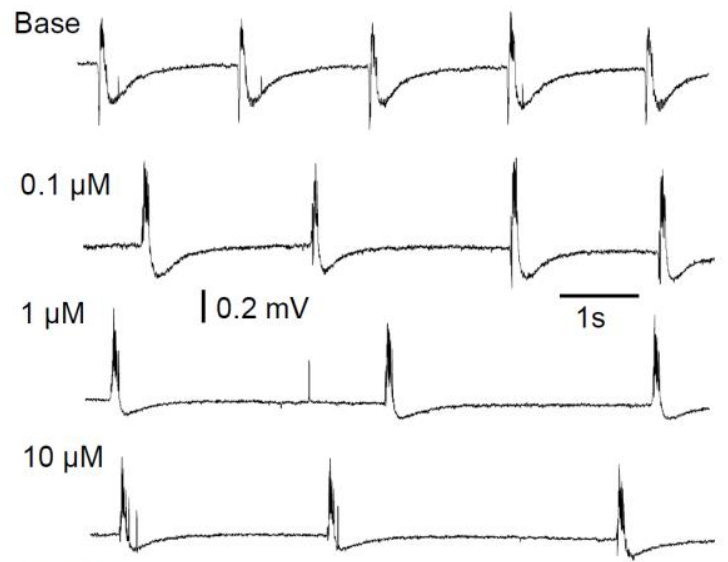

NO-711 b
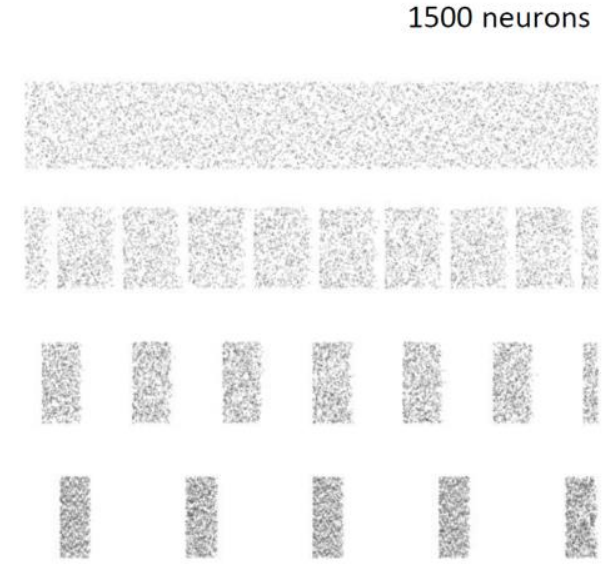
0005

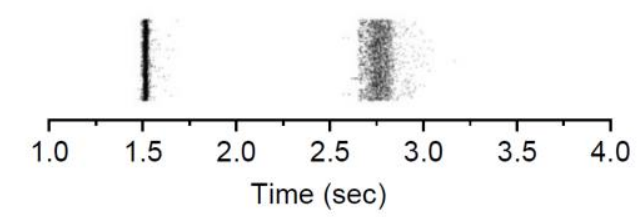

d

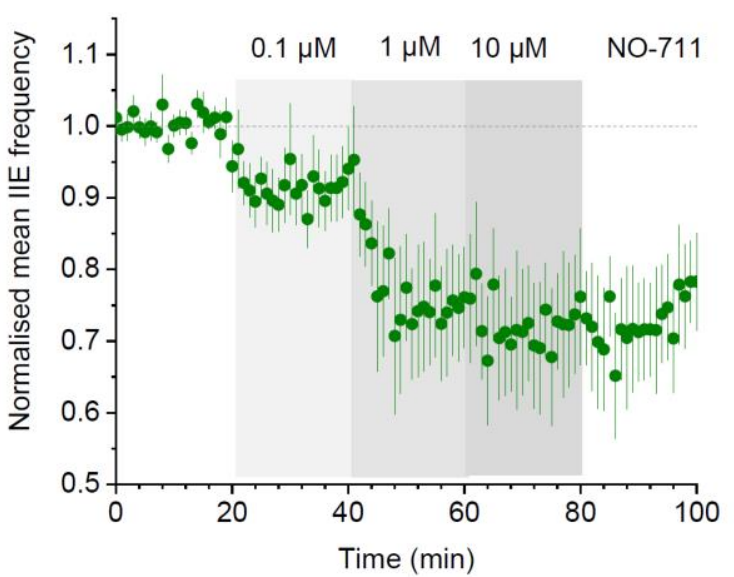

Figure 8. GABA uptake rate controls rhythmic activity of interneuronal networks. a, Modelled time course of $G_{\text {tonic }}$ at different GABA uptake rates, as indicated; key model parameters: cell number $N=1500$, intra-network peak synaptic conductance $G_{i i}=0.096 \mathrm{nS}$ $\mathrm{cm}^{-2}$; E-currents (Poisson series) with average synaptic conductance $g_{\mathrm{s}}=0.05 \mathrm{nS}$, decay constant tau $=3 \mathrm{~ms}$, and frequency $f_{s}=20 \mathrm{~Hz}$; $G_{\text {tonic }}$ reverse potential $V_{G A B A}=-53 \mathrm{mV}$, GABA release factor $A f=10^{-8} \mathrm{nS} \mathrm{cm} \mathrm{cm}^{-2} \mathrm{~ms}^{-1}, G_{\text {pump }}=0.004 \mathrm{~s}^{-1}$ (see Methods for further detail); $G_{\text {tonic }}$ scale (bottom panel) applies throughout. $\mathbf{b}$, Raster plots of interneuron spiking in the network, for the six cases shown in a; for presentation clarity, 6000 randomly selected spike events shown only, in each panel. c, Examples of interictal spiking recorded in slice, under basal conditions (Base), and during subsequent bath applications of the GABA uptake blocker NO711 at increasing concentrations, as indicated. d, Average frequency of interictal events (mean \pm s.e.m., $n=6$ slices), normalised to the baseline value, in baseline conditions and during NO711 application, as indicated. 
The waves of [GABA] e provide a volume-transmitted feedback signal that modulates neuronal firing. It was earlier reported that reducing $G_{\text {tonic }}$ from its basal (quiescent tissue) level boosts CA1 pyramidal cell firing while having little effect on interneuronal firing ${ }^{35}$, consistent with 'tonic' disinhibition of principal cells. However, increasing Gtonic above a certain level could have a similar effect on principal cells as it shunts interneuronal activity ${ }^{14}$, at which point the disinhibition of excitatory neurons again prevails leading to synchronized network discharges. The pattern of network activity and its predisposition to oscillations must therefore depend on the dynamics of Gtonic driven by [GABA] release, diffusion, and uptake.

\section{[GABA] dynamic range and activation of extrasynaptic receptors}

We find that during interictal discharges in hippocampal slices [GABA] e peaks at about 1.5-2 $\mu \mathrm{M}$. Calibration of the sniffer-patch sensor in acute slices indicates that [GABA] is maintained at 250-500 $\mathrm{nM}$ in nominally $\mathrm{Mg}^{2+}$-free aCSF. This concentration range is higher than $[\mathrm{GABA}]_{\mathrm{e}}$ in quiescent slices ( 100 nM), which we previously estimated using the same technique ${ }^{25}$, possibly explained by a lower spontaneous firing rate of interneurons in the higher $\left[\mathrm{Mg}^{2+}\right]$ concentrations. These estimates are generally in line with the $[G A B A]_{e}$ range reported earlier in hippocampal area $C A 1{ }^{36}$. Are these levels of $[\mathrm{GABA}]$ e compatible with peak values of Gtonic documented here using whole-cell recordings? One important prerequisite of having significant Gtonic is the presence of relatively low-affinity extrasynaptic GABARs that can stay in the sensitized state for prolonged periods of time. The well-described and broadly expressed high-affinity $\delta$ subunit-containing $\mathrm{GAB}_{\mathrm{A}} \mathrm{Rs}$ were found to become desensitized and almost irresponsive to GABA spillover when [GABA] reached $\sim 0.25 \mu \mathrm{M}^{37}$. Other receptor sub-types with $\mathrm{EC}_{50}$ in the range of $0.5-2 \mu \mathrm{M}$ include native metabotropic $\mathrm{GABA}_{\mathrm{B}}$ receptors in $L 2 / 3$ pyramidal neurons ${ }^{38}$, and receptors with subunits $\alpha 5 \beta 3 \gamma 2 L(0.5-7$ $\mu \mathrm{M})$, and $\alpha 1 \beta 2 \gamma 2 \mathrm{~L}(0.6-9 \mu \mathrm{M}){ }^{39}$. In general, it therefore appears plausible that the $[\mathrm{GABA}]_{\mathrm{e}}$ waves reported here find enough receptor targets to provide a significant dynamic range of $G_{\text {tonic }}{ }^{40}$.

\section{Mechanisms controlling [GABA]}

GABA that escapes from synaptic clefts (i.e., spills over) during interneuronal discharges is the most likely source of [GABA], although there have also been studies reporting GABA release from astroglia ${ }^{41-43}$. One plausible mechanism which could regulate $[G A B A]_{e}$ is the reversal mode of GABA transporters. Four GABA transporter types (GAT1-3 and BGT1), all showing potassium- and voltage-dependent kinetics, are expressed in both neurons and glial cells ${ }^{44-46}$. Intense excitatory activity, for instance during epileptiform bursts, can increase extracellular [ $\left.\mathrm{K}^{+}\right]$to $12-15 \mathrm{mM}^{47,48}$ and could, in principle, reverse GABA transport, thus generating GABA efflux ${ }^{49}$. As this type of condition is compatible with the present experimental settings, GABA transporters could potentially be a source of extracellular GABA coming from both neurons and glia. However, any significant rises of [GABA] e are likely to prevent the 
reversal transporter mode ${ }^{50}$. This argues against a significant contribution of nonsynaptic, transporter-mediated GABA release in conditions of our experiments. Finally, regulation of $[\mathrm{GABA}]_{\mathrm{e}}$ could arise from the Bestrophin $1 \mathrm{Ca}^{2+}$-dependent $\mathrm{Cl}^{-}$channel ${ }^{51}$ found predominantly in glial cells ${ }^{52}$. However, this mechanism is unlikely to play a role here, since the operation of this channel does not appear correlated with neuronal activity ${ }^{51}$.

\section{Constraining parameters of simulated neural networks}

Our study explored a well-established FS interneuron network model ${ }^{53}$ that incorporates $G_{\text {tonic }}$ driven by activity-dependent [GABA] e kinetics ${ }^{14,26}$. Whilst this model replicated key aspects of the experimentally documented interictal activity in the hippocampus, it had certain limitations. Firstly, we used a near-linear relationship between $[G A B A]_{e}$ and $G_{\text {tonic, }}$ whereas in reality it incorporates a more complex, supralinear dependence ${ }^{11,14}$. This might explain why the simulated $G_{\text {tonic }}$ dynamics appears more wave-like than the experimentally observed regular [GABA] rises.

Secondly, in the majority of simulations we implemented a simple stochastic excitatory input to mimic synaptic activity of pyramidal neurons, which is thus set independent of interneuronal activity. This approach was taken partly because the main aim of the present study was to establish whether and how the network activity of FS interneurons, on its own, could drive hippocampal oscillations. Nonetheless, a twinned simulated network containing both interneurons and principal cells displayed spiking behaviours that were fully consistent with the key features of interneuronal networks, and, more importantly, with experimental observations.

Finally, astroglia have also been considered a potentially important source of extracellular GABA due to reversal-mode release through GAT-1 and GAT-3 transporters. However, as pointed out above, such release can only occur at the lowest levels of [GABA] , hence out-of-phase with the interneuron activity, whereas our experimental observations combined indicate the opposite trend. Nonetheless, the role of astroglial GABA uptake must play an important role in pacing network rhythms, as suggested by both simulations and experiments that established a clear relationship between the GABA uptake rate and the frequency of epileptiform events.

\section{Interneurons and principal neurons in rhythm initiation}

Our sniffer-patch experiments, GABA imaging, and recordings of FS PV+ interneuron activity point to an increased tonic inhibition before interictal discharge onset. This observation invokes two possibilities: either interictal activity is due to the disinhibition of principal cells (due to the 'self-inhibition' of the interneuronal network or to depolarisation block) or interneuron firing itself drives interictal discharges. Our experiments reveal that synchronised interneuronal network activity can determine the timing of co-ordinated pyramidal cell bursts, confirming the earlier suggestion that interneurons provide local spatiotemporal control of pyramidal neurons and are critically important for generating such network rhythms ${ }^{54,55}$. At the same time, 
interneuronal activity per se can make an important contribution to epileptiform discharges.

Different areas of the brain display various combinations of physiological processes involving oscillations of interneuron networks. We would like to highlight two characteristic mechanisms behind such periodic activities, which may or may not depend on [GABA]e and Gtonic. Firstly, the so-called giant depolarising potentials (GDPs), which are network-driven wave-like events occurring during development ${ }^{56-58}$. The average GDP duration is around one second, with a fairly random spiking pattern involving 6-10 discharges per minute ${ }^{59,60}$. It has been suggested that GDPs are generated by synergistic activity of pyramidal cells and interneurons ${ }^{61}$. The second mechanism involves high-frequency (up to $200 \mathrm{~Hz}$ ) oscillations, referred to as hippocampal ripples ${ }^{62-64}$, and represent highly synchronous bursts of brain activity. In acute slices of ventral hippocampus, such ripples can occur in a periodic pattern, with a frequency of $7-14 \mathrm{~Hz}{ }^{65}$, similar to the theta-rhythm. These oscillatory patterns can thus arise purely from the interaction of excitatory and inhibitory neurons, a mechanism qualitatively different from interictal activity resulting from periodic fluctuations in [GABA]e and Gtonic.

\section{METHODS}

\section{Animal experimentation}

All experiments involving animals were carried out in accordance with the European Commission Directive (86/609/EEC) and the United Kingdom Home Office (Scientific Procedures) Act (1986), under the Home Office Project Licence PPL P2E0141 E1.

\section{Acute slice preparation}

In vitro electrophysiological recordings were performed in acute hippocampal slices prepared from 3- to 4-week-old male Sprague-Dawley rats (Harlan Laboratories Inc, Bicester, UK). Animals were kept under standard housing conditions with $12 \mathrm{~h}$ lightdark cycle and free access to food pellets and drinking water. After being sacrificed using an overdose of isoflurane, animals were decapitated, brains were rapidly removed, and hippocampi were dissected for slice preparation. Transverse hippocampal slices (350 $\mu$ m-thick) were cut with a Leica VT1200S vibratome (Germany) in an ice-cold sucrose-based solution containing (in $\mathrm{mM}$ ): sucrose (70), $\mathrm{NaCl}(80), \mathrm{KCl}$ (2.5), $\mathrm{MgCl}_{2}$ (7), $\mathrm{CaCl}_{2}$ (0.5), $\mathrm{NaHCO}_{3}$ (25), $\mathrm{NaH}_{2} \mathrm{PO}_{4}$ (1.25), glucose (22), bubbled continuously with $95 \% \mathrm{O}_{2}+5 \% \mathrm{CO}_{2}$ to yield a pH of 7.4 . Slices were allowed to recover in a sucrose-free artificial cerebrospinal fluid (aCSF) solution (in $\mathrm{mM}$ ): $\mathrm{NaCl}$ (119), $\mathrm{KCl}$ (2.5), $\mathrm{MgSO}_{4}$ (1.3), $\mathrm{CaCl}_{2}$ (2.5), $\mathrm{NaHCO}_{3}$ (26.2), $\mathrm{NaH}_{2} \mathrm{PO}_{4}$ (1), glucose (22), bubbled with $95 \% \mathrm{O}_{2}$ and $5 \% \mathrm{CO}_{2}$ in an interface chamber for at least 1 hour at room temperature before being transferred to a submerged recording chamber. 
Modified aCSF (nominally $0 \mathrm{mM} \mathrm{Mg}^{2+}$ and $5 \mathrm{mM} \mathrm{K}^{+}$, unless specified otherwise) was used to induce epileptiform activity. To facilitate the rapid generation of epileptiform discharges slices were perfused with the solution on both sides. All recordings were done at $32^{\circ} \mathrm{C}$. GABAB receptors were blocked in all experiments by $5 \mu \mathrm{M}$ CGP52432. Field potential recordings from stratum pyramidale were performed with 1-2 $\mathrm{M} \Omega$ glass electrodes filled with aCSF. Visualized whole-cell voltage-clamp recordings were performed from CA1 pyramidal neurons using an infrared differential interference contrast imaging system. Recording pipettes (3-5 M $\Omega$ ) were filled with internal solution, containing (in mM): Cs-methanesulfonate (120), HEPES (10), EGTA (0.2), NaCl (8), $\mathrm{MgCl}_{2}$ (0.2), Mg-ATP (2), Na-GTP (0.3), QX-314 Br- salt (5), pH 7.2, osmolarity 290 mOsm kg-1. GABAAR-mediated currents were recorded from neurons voltage clamped at $0 \mathrm{mV}$ or $+10 \mathrm{mV}$ (close to the reversal potential of glutamatergic currents). To further prevent the contribution of NMDA receptors MK801 $(1 \mu \mathrm{M})$ was included in the recording pipettes in acute slices experiments. Areas CA3 were cut off in the outsideout patch experiments, but not in other settings: this had no detectable effect on interneuronal network activity in area CA1.

Series resistance $\left(R_{s}\right)$ was monitored throughout the experiment using a $-5 \mathrm{mV}$ step command. Cells showing a $>20 \%$ change in $R_{s}$, or values $>25 \mathrm{M} \Omega$, or an unstable holding current, were rejected. Recordings were obtained using a MultiClamp 700B amplifier (Molecular Devices, CA, USA), filtered at $4 \mathrm{kHz}$, digitized and sampled through an AD converter Digidata 1550 (Molecular Devices) or NI PCI-6221M (National Instruments) at $10 \mathrm{kHz}$ and stored on a PC. Data acquisition and off-line analysis were performed using WinEDR 3.0.1 (University of Strathclyde, Glasgow, UK) and Clampfit 10.0 (Molecular Devices Corporation, USA) software, or Femtonics MES (Femtonics, Budapest) and custom-made Matlab (Mathworks) scripts running in Matlab r2020b.

\section{Outside-out patch recordings}

Single channel GABA $A_{A}$-mediated currents were recorded in the presence of $0.1 \mu \mathrm{M}$ CGP-55845 as detailed earlier 17, 25. Outside-out patches were pulled from dentate granule cells, and recordings were performed in voltage-clamp mode at $33-35^{\circ} \mathrm{C}$. For membrane patches held at $-70 \mathrm{mV}$, the recording electrode solution contained (in $\mathrm{mM}$ ): $120.5 \mathrm{CsCl}, 10 \mathrm{KOH}$-HEPES, $10 \mathrm{BAPTA}, 8 \mathrm{NaCl}, 5 \mathrm{QX}-314-\mathrm{Br}^{-}$salt, $2 \mathrm{Mg}-\mathrm{ATP}$, $0.3 \mathrm{Na}-\mathrm{GTP}$; $\mathrm{pH} 7.2,295 \mathrm{mOsm} \mathrm{kg}^{-1}$. For membrane patches held at $0 \mathrm{mV}$, the recording electrode solution contained (in $\mathrm{mM}$ ): $120 \mathrm{Cs}$-methanesulfonate, $10 \mathrm{HEPES}$, 0.2 EGTA, $8 \mathrm{NaCl}, 0.2 \mathrm{MgCl}_{2}$, 2 Mg-ATP, 0.3 Na-GTP, 5 QX-314-Br- salt; pH 7.2, 290 $\mathrm{mOsm} \mathrm{kg}{ }^{-1}$. Recordings were made using MultiClamp 700B amplifier (Molecular Devices, CA, USA); signals were digitized at $10 \mathrm{kHz}$. The patch pipette resistance was 5-7 M $\Omega$. In experiments where GABA EC 50 was determined, we used a $\theta$-glass application pipette with $\sim 200 \mu \mathrm{m}$ tip diameter attached to a micromanipulator. The pipette position was controlled by a piezoelectric element (each switch took 50-100 $\mu \mathrm{s})$ to allow rapid solution exchange. One pipette channel was filled with the bath 
aCSF solution; another channel had aCSF with varying concentrations of GABA. The flow rate was driven by gravity 66 .

\section{Analysis of single-channel recordings}

The frequency of GABAAR channel openings was calculated as $N / \Delta t$, where $N$ is the number of openings and $\Delta t$ is the time of recording. $\mathrm{N}$ was counted using a detection threshold of $1.5 \mathrm{pA}$ abive the mean baseline value and a minimum opening time of $>0.2 \mathrm{~ms}$. Single channel conductance was calculated as $G=I /\left(V_{\text {rev }}-V_{\text {hold }}\right)$, where $I$ stands for receptor-mediated current, and $V_{\text {rev }}$ - calculated chloride reversal potential. The average charge transfer through receptors was calculated as $Q=G \times N / \Delta t$. The concentration-effect plot was fitted with the Hill equation $E=\frac{[C]^{n}}{K_{d}{ }^{n}+[C]^{n}}$, where $E$ stands for the percentage of maximal charge transfer, $C$ concentration of GABA, $K_{d}$ microscopic dissociation constant (equal to $\mathrm{EC}_{50}$ ), and $n$ Hill's coefficient.

\section{Organotypic slice culture preparation and biolistic transfection of iGABASnFR}

Organotypic hippocampal slice cultures were prepared and grown with modifications to the interface culture method from P6-8 Sprague-Dawley rats as previously described 31. In brief, $300 \mu \mathrm{m}$ thick, isolated hippocampal brain slices were sectioned using a Leica VT1200S vibratome in ice-cold sterile slicing solution consisting of (in mM) Sucrose 105, $\mathrm{NaCl} 50, \mathrm{KCl} 2.5, \mathrm{NaH}_{2} \mathrm{PO}_{4}$ 1.25, $\mathrm{MgCl}_{2} 7, \mathrm{CaCl}_{2} \mathrm{0.5}$, Ascorbic acid 1.3, Sodium pyruvate 3, $\mathrm{NaHCO}_{3} 26$ and Glucose 10. Following washes in culture media consisting of 50\% Minimal Essential Media, 25\% Horse Serum, 25\% Hanks Balanced Salt solution, $0.5 \%$ L-Glutamine, $28 \mathrm{mM}$ Glucose and the antibiotics penicillin $(100 \mathrm{U} / \mathrm{ml})$ and streptomycin $(100 \mu \mathrm{g} / \mathrm{ml})$, three to four slices were transferred onto each $0.4 \mu \mathrm{m}$ pore membrane insert (Millicell-CM, Millipore, UK). Cultures were then maintained at $37^{\circ} \mathrm{C}$ in $5 \% \mathrm{CO}_{2}$ and fed by medium exchange every $2-3$ days for a maximum of 21 days in vitro (DIV). At 5DIV cultures were treated overnight with $5 \mu \mathrm{M}$ cytosine arabinoside (Ara-C, a poison inhibitor of DNA and RNA polymerases) to reduce glial reaction following biolistic transfection and returned to standard culture media at 6DIV. At 8DIV cultures were shot with 1.6 micron Gold micro-carriers coated with $30 \mu \mathrm{g}$ of hSyn.iGABASnFR.F102G plasmid using the Helios gene-gun system (Bio-Rad). Following transfection cultures remained for 5-10 days before experiments were carried out.

\section{Imaging of interictal epileptiform activity associated extracellular GABA transients}

For imaging of iGABASnFR transients associated with interictal activity, organotypic slice cultures were cut from their membrane insert and transferred to the stage of a Femtonics Femto3D-RC imaging system (Femtonics, Budapest) integrated with patchclamp electrophysiology and linked on the same light path to two femtosecond pulse 
lasers MaiTai (SpectraPhysics-Newport) with independent shutter and intensity control as previously described ${ }^{31}$. Slices were continuously perfused with bicarbonate based artificial cerebrospinal fluid (aCSF) equilibrated with $95 \% \mathrm{O}_{2}$ and $5 \% \mathrm{CO}_{2}$ at $32-34{ }^{\circ} \mathrm{C}$ using a gravity driven perfusion system (flow rate $3-4 \mathrm{ml} / \mathrm{min}$ ). aCSF solution contained (in mM): $120 \mathrm{NaCl}, 10$ glucose, $2.5 \mathrm{KCl}, 1.3 \mathrm{MgSO}_{4}, 1 \mathrm{NaH}_{2} \mathrm{PO}_{4}, 25 \mathrm{NaHCO}_{3}, 2 \mathrm{CaCl}_{2}$ with osmolality of $300 \pm 5$ mOsm kg-1. Biolistic transfection of iGABASnFR resulted in sparse labelling of neurones in the CA1 and CA3 regions making identification of iGABASnFR expressing cells using two-photon imaging $\left(\lambda_{x}^{2 p}=910 \mathrm{~nm}\right)$ unambiguous. Following identification, a glass field recording electrode filled with standard aCSF was placed within $\sim 30 \mu \mathrm{m}$ of the cell of interest and interictal activity was then initiated by exchanging the extracellular solution for a $0 \mathrm{mM} \mathrm{Mg}^{2+}$ aCSF (otherwise the same composition). The local field potential was then monitored until stable interictal bursting was observed, at which point curved frame-scan regions of interest following the somatic membrane were chosen for scanning (see Fig. 2a for illustration). To avoid photodamage, scan duration was limited to 9 second epochs which were repeated with a one-minute intervals until a minimum of 10 events were captured. For comparison of interictal event timing and associated iGABASnFR transients; interictal event peak times were first identified by the findpeaks function in MATLAB and were accepted for further analysis where single peaks with a minimum width of $20 \mathrm{~ms}$ and amplitude $>5 \times$ standard deviation (SD) of baseline noise could be detected. The associated iGABASnFR $\triangle F / F$ peak was then identified (again using findpeaks) within a manually adjusted window $-750 \mathrm{~ms}$ to $+2 \mathrm{~s}$ (extremes between cells/slices) over the peak times identified for the interictal event, with a minimum $>2.5 \times$ SD baseline and minimum width of $50 \mathrm{~ms}$. Events within that window were then analysed where a clear and stable iGABASnFR $\triangle F / F 50 \mathrm{~ms}$ length baseline, rise and visible decay associated could be observed. The rise to peak from baseline was then fitted by polynomial regression and the 20\% iGABASnFR peak time extrapolated from the fit and used to calculate iGABASnFR transient-interictal event lag times.

\section{Targeted cell-attached recordings and optogenetic experiments.}

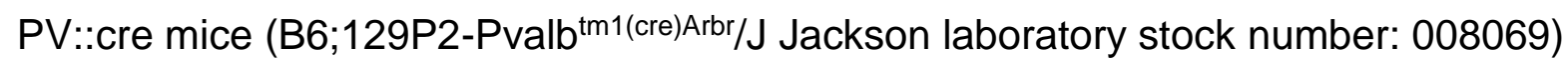
were crossed with Ai9 or Ai32 mouse line, which has floxed-stop tdTomato (B6.CgGt(ROSA)26Sortm9(CAG-tdTomato)Hze/J Jackson laboratory stock number: 007909) or EYFP-tagged excitatory opsin channelrhodopsin-2-H134R (B6;129S-

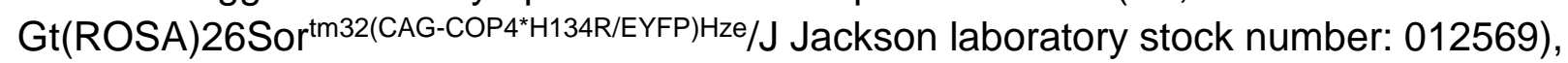
to produce animals expressing tdTomato or channelrhodopsin-2 (ChR2) in parvalbumin-positive $\left(\mathrm{PV}^{+}\right)$interneurons throughout the brain ${ }^{67}$. Animals were kept under standard housing conditions with $12 \mathrm{~h}$ light-dark cycle and free access to food pellets and drinking water. Hippocampal slices were prepared from mice of both sexes aged between postnatal day 25 and 50 for the electrophysiology section, but under low light intensity to minimise any adverse effect due to activation of ChR2. Field potential recordings from stratum pyramidale and visualized whole-cell voltage-clamp recordings were performed from CA1 pyramidal neurons using infrared differential 
interference contrast imaging system as in the electrophysiology section. For the dual cell-attached recordings (Fig. 5), borosilicate glass pipettes (3-5 M $\Omega$ ) filled with aCSF and containing Alexa Fluor $488(50 \mu \mathrm{M})$ were used and the whole-cell recording pipettes was containing the standard Cs-methylsulfonate cited above with the addition of Alexa Fluor 488. In addition, the slices were superfused with aCSF with $0 \mathrm{mM} \mathrm{Mg} \mathrm{Mg}^{2+}$ and $10 \mathrm{mM} \mathrm{K}^{+}$as well as APV $(50 \mu \mathrm{M})$, NBQX (10 $\left.\mu \mathrm{M}\right)$ and CGP52432 (1 $\left.\mu \mathrm{M}\right)$.

Cells showing a series resistance $>25 \mathrm{M} \Omega$, or an unstable holding current, were rejected. Recordings were obtained using a MultiClamp 700B amplifier (Molecular Devices, CA, USA), filtered at $2-4 \mathrm{kHz}$ and digitized at $10 \mathrm{kHz}$. Data acquisition and off-line analysis were performed using WinEDR 3.0.1 (University of Strathclyde, Glasgow, UK) and Clampfit 10.0 (Molecular Devices Corporation, USA) softwares as well as MATLAB and Python custom-scripts.

Wide-field illumination of the CA1 region of the hippocampus was delivered through a 20x water immersion objective (Olympus). PV tdTomato-positive cells were visualised with $590 \mathrm{~nm}$ wavelength and ChR2 was activated by blue light (wavelength $470 \mathrm{~nm}$ ) generated by pE-2 LED illumination system (CoolLED); light intensity under the objective was in the range of $5-10 \mathrm{~mW}$. For the ramp stimulation protocol, a train of 1 $\mathrm{s}$ ramps was delivered at on average $\sim 0.3 \mathrm{~Hz}$ ranging from 0.12 to $0.33 \mathrm{~Hz}$.

\section{Interictal discharges analysis}

The detection of interictal spikes was done using custom-made python script. Local field electrophysiological trace was first downsampled to $1 \mathrm{kHz}$ and filtered using a Butterworth bandpass filter from 1 to $50 \mathrm{~Hz}$. The onset of interictal discharges was determined by using a detection threshold on the second derivative of the local field potential recording. The threshold corresponded to a minimum of 5 SDs above baseline noise (taking from the beginning of each trace). For the probability of inducing an interictal events with $1 \mathrm{~ms}$ light pulse stimulation, a window of $100 \mathrm{~ms}$ poststimulation was considered for the detection of an event. Concerning the ramp stimulation, the window was between $500 \mathrm{~ms}$ after the start of the ramp and $100 \mathrm{~ms}$ post-stimulation.

\section{Materials and statistical analysis}

Glutamate and GABA receptor blockers were purchased from Tocris Cookson (Bristol, UK) and Ascent Scientific (North Somerset, UK). Two-tailed paired and unpaired Student's $t$-test were used for statistical analysis of electrophysiological data. Mean difference was considered significant at the null-hypothesis rejection level of $p<0.05$. Statistical summary data are shown as mean \pm s.e.m. unless specified otherwise.

\section{Modelling: Interneurons and pyramidal neurons networks}


The interneurons and pyramidal neuron (principal cell, PC) networks were simulated on a digital platform ARACHNE with remotely-controlled parallel computing ${ }^{27}$. Similar to the previously explored non-hierarchical networks ${ }^{14}$, the present one featured circular connectivity (Fig. 3a), which helped (a) exclude edge effects, (b) equalise cell contribution, and (c) represent the size by a single parameter, network radius $R$. In individual simulated cells, the channel kinetics were typical of hippocampal fast-spiking basket interneurons ${ }^{53}$. Other incorporated biophysical mechanisms, such as ion channel flux, GABA transporter, synaptic and GABA tonic current were in accord with experimental findings ${ }^{68-70}$, as detailed below. The time course of GABAergic synaptic conductance followed the function $y(t)=G_{i i}\left(\exp \left(-t / \tau_{1}\right)-\exp \left(-t / \tau_{2}\right)\right)$ where $y(t)$ is the synaptic conductance at time $t, \tau 1$ is the rise time constant, $\tau 2$ (termed tau elsewhere) is the decay time constant, $G_{i i}$ is the peak conductivity $\left(\mathrm{mS} \mathrm{cm}^{-2}\right)$ of synapses.

A network of interneurons and pyramidal cells was simulated using previously published models ${ }^{68,69}$ with minor modifications of the internal parameters, and a critical addition of the dynamic Gtonic, as explained below, with the Gtonic range in interneurons twice that of pyramidal cells, in accord with experimental data ${ }^{14}$; the basic cell-circuit model used in the present study was obtained from ModelDB (accession no. 138421). Briefly, the modelled circular network consisted of $N$ fastspiking interneurons ( $I$-cells) and $M$ pyramidal cells ( $E$-cells). Each cell was modelled as a single compartment using standard Hodgkin-Huxley formalism. The GABAAR reversal potential $V_{G A B A}$ was set between $-55 \mathrm{mV}$ and $-72 \mathrm{mV}$, as specified. Unless specified otherwise, the network featured three connection types: $I-I, I-E$, and $E-I$, with release probabilities values $P_{E-E}=0.5, P_{E-I}=0.5$, and $P_{I-I}=1.0$, respectively. The spatial synaptic connection density was defined as the matrix $W_{X Y}(i, j)$ with the Gaussian distribution centred at a given neuron, so that

$W_{X Y}(i, j)=W_{X Y \max } \exp \left(-\frac{1}{2}\left(\frac{\max (i, j)-1}{\sigma_{x y}}\right)^{2}\left(\frac{j-1}{\max (j)}-\frac{i-1}{\max (i)}\right)^{2}\right)$

The model consists in bell-shaped strength and Gaussian density of connections. Synaptic conductance matrices of this model are with following strength coefficient $W_{e e, \max }=2, W_{i i, \max }=0.8, W_{e i, \max }=0.9 ; W_{i e, \max }=0.3$, and spatial SD: $\sigma_{\text {ee }}=10 \mu \mathrm{m}, \sigma_{\mathrm{ei}}=12.5$ $\mu \mathrm{m}, \sigma_{\mathrm{ie}}=8 \mu \mathrm{m}$ and $\sigma_{\mathrm{ii}}=5 \mu \mathrm{m}$. The size (radius) of interneuron and principal cell network was set at 250 and $200 \mu \mathrm{m}$, respectively, with the signal propagation speed at $0.1 \mu \mathrm{m} \mathrm{ms}{ }^{-1}$.

Simulations were performed using ARACHNE on the Amazon AWS cloud computing (cluster c5.large, tolerance $10^{-5}$, time step $0.02 \mathrm{~ms}$ ). Random generator use32BitRng (MATLAB) was set to generate a delta correlated white noise for any stochastic processes. The initial voltage of interneurons in the network was set uniformly randomly, between -73 and $-67 \mathrm{mV}$. Mechanisms of synaptic plasticity were excluded from the basic model of the neural network. 
The network synchronization parameter $k(\tau)$, which was also used for the experiment in PV+ cell pairs (Fig. 5), was calculated as an average of all coefficients $k_{i, j}(\tau)$ for each pair of neurons $(i, j)$. The time window of synchronization was divided into bins so that $\tau=0.1 / f_{m}$ where $f_{m}$ is the network-average spiking frequency (see below). In each bin, an action potential was represented in a binary format (yes-no series). Next, $k_{i, j}(\tau)$ was calculated for each pair of neurons for the time bin $\tau$ using the formula:

$k_{i j}(\tau)=\frac{\sum_{l=1}^{K} X(l) Y(l)}{\sqrt{\sum_{l=1}^{K} X(l) \sum_{l=1}^{K} Y(l)}}$

where $X$ and $Y$ are the binary series of the $i$ th and $j$ th cells, respectively, $l$ is the bin number; thus, $X(l)$ and $Y(l)$ are either 0 or 1 depending on having a spike (1) or no spike $(0)$ event for $i$ th or $j$ th during the $l$ th bin, and $K$ is the total number of bins.

\section{Modelling: Equations for the tonic GABA conductance}

$[\mathrm{GABA}]_{\mathrm{e}}$ activates extrasynaptic $\mathrm{GABA} A \mathrm{Rs}$, which generate Gtonic. Thus, modelled cells generate non-specific tonic current: $I_{\text {tonic }}=G_{\text {tonic }}\left(V_{G A B A}-V_{m}\right)$, where $V_{\mathrm{GABA}}$ is GABA reversal potential, and $V_{m}$ is the membrane potential. $G_{\text {tonic }}$ depends on the interneuron network firing frequency, which in turn generates [GABA] increments, in accord with:

$\frac{d G_{\text {tonic }}}{d t}=N_{i} A_{f}\left(f_{m}[t-d t]+f_{b}\right)-G_{\text {pump }}\left(G_{\text {tonic }}-G_{\text {basal }}\right)$.

with initial $G_{\text {tonic }}(0)=G_{\text {basal }}$ and $f_{m}$ is calculated as the total of cell-generated spikes over time $T$ :

$$
f_{m}=\frac{1}{T N_{i}} \sum_{i=1}^{N_{i}} A P_{i}(T, t) .
$$

Other parameters were: $N\left(\right.$ or $\left.N_{i}\right)$, total number of neurons in the network; $A_{f}(0.01 \mathrm{nS}$ $\mathrm{cm}^{-2} \mathrm{~ms}^{-1}$, unless specified otherwise), tonic GABA conductance resulting from a single AP over $100 \mathrm{~ms}$; Gpump (values as indicated), the GABA uptake rate, $G_{\text {basal }}=$ $0.1 \mathrm{mS} \mathrm{cm}^{-2}$, [GABA] $]_{\mathrm{e}}$ value at which GABA transporters reverse, $f_{b}=0.1 \mathrm{~Hz}$, basal average network spiking frequency at rest; and $d t$, a delay between neurotransmitter release and activation of extrasynaptic receptors ( $d t$ was $<1 \mathrm{~ms}$ throughout).

In our simulations, we assumed that $G_{\text {tonic }}$ linearly depends on [GABA]e:

$G_{\text {tonic }}=\alpha[G A B A]_{e}$. This assumption provides a good approximation $[\mathrm{GABA}]_{\mathrm{e}}<1-2 \mu \mathrm{M}$, as in this case it falls into the near-linear approximation of the charge transfer versus $[\mathrm{GABA}]_{\mathrm{e}}$ relationship (Fig. 1e). The Gtonic dynamics was therefore determined by the time course of [GABA] e, which is represented by quasi-instantaneous release followed by uptake. The latter was set in accord with previous estimates of the GAT-1 kinetics ${ }^{50}$, so that solution of equation (1) for constant $f_{m}$ was 


$$
G_{\text {tonic }}(t)=e^{-t_{p} t} G_{\text {basal }}+\frac{A_{f} f_{m} N_{i}}{t_{p}} e^{-t_{p} t}\left(e^{t_{p} t}-1\right),
$$

where $t_{p}$ is $0.02-0.1 \mathrm{~ms}^{-1}$ and $A_{f}$ is the Gbasal $/$ Gtonic ratio at steady-state for a given frequency $f_{m}: A_{f}=\frac{K G_{i n i t} t_{p}}{f_{m} N_{i}}$, where $2<K<4$. Thus, $A_{f}$ was a scaling factor for $[\mathrm{GABA}] \mathrm{e}$ action.

\section{Modelling: $[G A B A]_{e}$ dynamics}

The dynamics of $[\mathrm{GABA}]_{\mathrm{e}}$ was estimated from the cell-spiking raster plot, with individual action potentials releasing GABA at a rate of $0.5 \mu \mathrm{M} \mathrm{ms}^{-1}$ of GABA $(\sim 3000$ molecules) in an extracellular volume of $20 \mu \mathrm{m}$ radius, the average distance between neighbouring neurons of the network. [GABA] decays due to GABA uptake and diffusion, a typical constant of $0.004 \mu \mathrm{M} \mathrm{ms}^{-1}$ (ref. ${ }^{50}$ ), unless specified otherwise.

\section{Modelling: Code availability}

ARACHNE is available with explanatory documentation at www.neuroalgebra.com. The program is made available under MIT license. ARACHNE is written in a way that allows users to run it on any remote platforms. Files with a set of initial parameters for reproducing the results compatible with ARACHNE can be provided upon request. For any computations we provided the exe file of ARACHNE, but also the initial code on https://github.com/LeonidSavtchenko/Arachne.

\section{ACKNOWLEDGEMENTS}

This work was supported by Epilepsy Research UK, MRC, Wellcome Trust Principal Fellowship, ERC Advanced Grant. Optimization and parallelization of ARACHNE algorithms for extended neural network simulations was provided by Andrey Galkin and team at AMC Bridge (Waltham, MA).

\section{AUTHOR CONTRIBUTIONS}

I.P., L.P.S. and D.A.R narrated the study; I.P. and V.M. designed and carried out electrophysiological and optogenetic studies; N.C. carried out iGABASnFR imaging experiments designed and analysed by T.P.J; S.S. carried out sniffer-patch experiments; L.P.S. designed and carried out network modelling studies and data analyses; J.S.M. and L.L.L. developed iGABASnFR imaging protocols; D.M.K. and M.C.W. designed optogenetic experiments and assisted with analyses; D.A.R. 
designed selected experiments and simulations and wrote the manuscript with I.P. and L.P.S, which was subsequently contributed to by V.M. and all other authors.

\section{COMPETING INTEREST STATEMENT}

The authors declare no competing interests

\section{REFERENCES}

1. van der Zeyden, M., Oldenziel, W.H., Rea, K., Cremers, T.I. \& Westerink, B.H. Microdialysis of GABA and glutamate: analysis, interpretation and comparison with microsensors. Pharmacol Biochem Behav 90, 135-147 (2008).

2. Overstreet, L.S. \& Westbrook, G.L. Synapse density regulates independence at unitary inhibitory synapses. J Neurosci 23, 2618-2626 (2003).

3. Scanziani, M. GABA spillover activates postsynaptic GABA(B) receptors to control rhythmic hippocampal activity. Neuron 25, 673-681 (2000).

4. Loo, D.D.F., Eskandari, S., Boorer, K.J., Sarkar, H.K. \& Wright, E.M. Role of Clin electrogenic $\mathrm{Na}+-$ coupled cotransporters GAT1 and SGLT1. J. Biol. Chem. 275, 37414-37422 (2000).

5. Bicho, A. \& Grewer, C. Rapid substrate-induced charge movements of the GABA transporter GAT1. Biophys J 89, 211-231 (2005).

6. MacAulay, N., Meinild, A.K., Zeuthen, T. \& Gether, U. Residues in the extracellular loop 4 are critical for maintaining the conformational equilibrium of the gamma-aminobutyric acid transporter-1. J. Biol. Chem. 278, 28771-28777 (2003).

7. Glykys, J. \& Mody, I. The main source of ambient GABA responsible for tonic inhibition in the mouse hippocampus. J Physiol 582, 1163-1178 (2007).

8. Marchionni, I. \& Maccaferri, G. Quantitative dynamics and spatial profile of perisomatic GABAergic input during epileptiform synchronization in the CA1 hippocampus. J Physiol 587, 5691-5708 (2009).

9. Brickley, S.G., Revilla, V., Cull-Candy, S.G., Wisden, W. \& Farrant, M. Adaptive regulation of neuronal excitability by a voltage-independent potassium conductance. Nature 409, 88-92 (2001).

10. Pavlov, I., Savtchenko, L.P., Kullmann, D.M., Semyanov, A. \& Walker, M.C. Outwardly rectifying tonically active GABAA receptors in pyramidal cells modulate neuronal offset, not gain. J Neurosci 29, 15341-15350 (2009).

11. Song, I., Savtchenko, L. \& Semyanov, A. Tonic excitation or inhibition is set by $\operatorname{GABA}(A)$ conductance in hippocampal interneurons. Nat Commun 2, 376 (2011).

12. Scimemi, A., Semyanov, A., Sperk, G., Kullmann, D.M. \& Walker, M.C. Multiple and Plastic Receptors Mediate Tonic GABA<sub $>A</$ sub $>$ Receptor Currents in the Hippocampus. The Journal of Neuroscience 25, 10016-10024 (2005).

13. Mann, E.O. \& Mody, I. Control of hippocampal gamma oscillation frequency by tonic inhibition and excitation of interneurons. Nat Neurosci 13, 205-212 (2010).

14. Pavlov, I., et al. Tonic GABAA conductance bidirectionally controls interneuron firing pattern and synchronization in the CA3 hippocampal network. Proc Natl Acad Sci U S A 111, 504-509 (2014). 
15. Pouille, F. \& Scanziani, M. Enforcement of temporal fidelity in pyramidal cells by somatic feed-forward inhibition. Science 293, 1159-1163 (2001).

16. Pavlov, I., Scimemi, A., Savtchenko, L., Kullmann, D.M. \& Walker, M.C. I(h)mediated depolarization enhances the temporal precision of neuronal integration. Nat Commun 2, 199 (2011).

17. Sylantyev, S., Savtchenko, L.P., O'Neill, N. \& Rusakov, D.A. Extracellular GABA waves regulate coincidence detection in excitatory circuits. J. Physiol. 598, 40474062 (2020).

18. Karlocai, M.R., et al. Physiological sharp wave-ripples and interictal events in vitro: what's the difference? Brain 137, 463-485 (2014).

19. Miri, M.L., Vinck, M., Pant, R. \& Cardin, J.A. Altered hippocampal interneuron activity precedes ictal onset. Elife 7 (2018).

20. Librizzi, L., et al. Interneuronal Network Activity at the Onset of Seizure-Like Events in Entorhinal Cortex Slices. J. Neurosci. 37, 10398-10407 (2017).

21. Chang, M., et al. Brief activation of GABAergic interneurons initiates the transition to ictal events through post-inhibitory rebound excitation. Neurobiol Dis 109, 102116 (2018).

22. Huguenard, J.R. \& McCormick, D.A. Simulation of the currents involved in rhythmic oscillations in thalamic relay neurons. J Neurophysiol 68, 1373-1383 (1992).

23. Marvin, J.S., et al. A genetically encoded fluorescent sensor for in vivo imaging of GABA. Nature Methods 16, 763-770 (2019).

24. Isaacson, J.S., Solis, J.M. \& Nicoll, R.A. Local and Diffuse Synaptic Actions of Gaba in the Hippocampus. Neuron 10, 165-175 (1993).

25. Wlodarczyk, A.I., et al. GABA-independent GABAA receptor openings maintain tonic currents. J Neurosci 33, 3905-3914 (2013).

26. Savtchenko, L.P. \& Rusakov, D.A. Regulation of rhythm genesis by volumelimited, astroglia-like signals in neural networks. Philos Trans $R$ Soc Lond B Biol Sci 369 (2014).

27. Aleksin, S.G., Zheng, K., Rusakov, D.A. \& Savtchenko, L.P. ARACHNE: A neural-neuroglial network builder with remotely controlled parallel computing. PLoS Comput Biol 13, e1005467 (2017).

28. Sylantyev, S. \& Rusakov, D.A. Sub-millisecond ligand probing of cell receptors with multiple solution exchange. Nature Protocols 8, 1299-1306 (2013).

29. Armbruster, M., Dulla, C.G. \& Diamond, J.S. Effects of fluorescent glutamate indicators on neurotransmitter diffusion and uptake. Elife 9 (2020).

30. Kopach, O., Zheng, K.Y. \& Rusakov, D.A. Optical monitoring of glutamate release at multiple synapses in situ detects changes following LTP induction. Molecular Brain 13 (2020).

31. Jensen, T.P., et al. Multiplex imaging relates quantal glutamate release to presynaptic $\mathrm{Ca} 2+$ homeostasis at multiple synapses in situ. Nature Communications 10, 1414 (2019).

32. Savtchenko, L., Megalogeni, M., Rusakov, D.A., Walker, M.C. \& Pavlov, I. Synaptic GABA release prevents GABA transporter type-1 reversal during excessive network activity. Nat Commun 6, 6597 (2015).

33. Fasoli, D., Cattani, A. \& Panzeri, S. Transitions between asynchronous and synchronous states: a theory of correlations in small neural circuits. J Comput Neurosci 44, 25-43 (2018). 
34. Rusakov, D.A. The role of perisynaptic glial sheaths in glutamate spillover and extracellular $\mathrm{Ca}^{2+}$ depletion. Biophys J 81, 1947-1959 (2001).

35. Semyanov, A., Walker, M.C. \& Kullmann, D.M. GABA uptake regulates cortical excitability via cell type-specific tonic inhibition. Nat Neurosci 6, 484-490 (2003).

36. Farahmandfar, M., Zarrindast, M.R., Kadivar, M., Karimian, S.M. \& Naghdi, N. The effect of morphine sensitization on extracellular concentrations of GABA in dorsal hippocampus of male rats. European journal of pharmacology 669, 66-70 (2011).

37. Bright, D.P., et al. Profound Desensitization by Ambient GABA Limits Activation of $\delta$-Containing GABA $<$ sub $>A</$ sub $>$ Receptors during Spillover. The Journal of Neuroscience 31, 753-763 (2011).

38. Wang, Y., Neubauer, F.B., Luscher, H.R. \& Thurley, K. GABAB receptordependent modulation of network activity in the rat prefrontal cortex in vitro. Eur $J$ Neurosci 31, 1582-1594 (2010).

39. Lagrange, A.H., Hu, N. \& Macdonald, R.L. GABA beyond the synapse: defining the subtype-specific pharmacodynamics of non-synaptic GABAA receptors. $J$ Physiol 596, 4475-4495 (2018).

40. Scimemi, A., Semyanov, A., Sperk, G., Kullmann, D.M. \& Walker, M.C. Multiple and plastic receptors mediate tonic GABAA receptor currents in the hippocampus. J Neurosci 25, 10016-10024 (2005).

41. Lee, M., McGeer, E.G. \& McGeer, P.L. Mechanisms of GABA release from human astrocytes. Glia 59, 1600-1611 (2011).

42. Heja, L., et al. Glutamate uptake triggers transporter-mediated GABA release from astrocytes. PLoS One 4, e7153 (2009).

43. Le Meur, K., Mendizabal-Zubiaga, J., Grandes, P. \& Audinat, E. GABA release by hippocampal astrocytes. Front Comput Neurosci 6, 59 (2012).

44. Borden, L.A. GABA transporter heterogeneity: pharmacology and cellular localization. Neurochemistry international 29, 335-356 (1996).

45. Gadea, A. \& Lopez-Colome, A.M. Glial transporters for glutamate, glycine, and GABA: II. GABA transporters. Journal of neuroscience research 63, 461-468 (2001).

46. Conti, F., et al. Neuronal and glial localization of GAT-1, a high-affinity gammaaminobutyric acid plasma membrane transporter, in human cerebral cortex: with a note on its distribution in monkey cortex. The Journal of comparative neurology 396, 51-63 (1998).

47. Krnjevic, K., Morris, M.E. \& Reiffenstein, R.J. Changes in extracellular Ca2+ and $\mathrm{K}+$ activity accompanying hippocampal discharges. Canadian journal of physiology and pharmacology 58, 579-582 (1980).

48. Somjen, G.G. \& Giacchino, J.L. Potassium and calcium concentrations in interstitial fluid of hippocampal formation during paroxysmal responses. Journal of neurophysiology 53, 1098-1108 (1985).

49. Wu, Y., Wang, W. \& Richerson, G.B. GABA Transaminase Inhibition Induces Spontaneous and Enhances Depolarization-Evoked GABA Efflux via Reversal of the GABA Transporter. The Journal of Neuroscience 21, 2630-2639 (2001).

50. Savtchenko, L., Megalogeni, M., Rusakov, D.A., Walker, M.C. \& Pavlov, I. Synaptic GABA release prevents GABA transporter type-1 reversal during excessive network activity. Nature Communications 6, 6597 (2015).

51. Lee, S., et al. Channel-mediated tonic GABA release from glia. Science (New York, N.Y.) 330, 790-796 (2010). 
52. Oh, S.J. \& Lee, C.J. Distribution and Function of the Bestrophin-1 (Best1) Channel in the Brain. Experimental neurobiology 26, 113-121 (2017).

53. Wang, X.J. \& Buzsaki, G. Gamma oscillation by synaptic inhibition in a hippocampal interneuronal network model. J Neurosci 16, 6402-6413 (1996).

54. Traub, R.D., Jefferys, J.G. \& Whittington, M.A. Functionally relevant and functionally disruptive (epileptic) synchronized oscillations in brain slices. Adv Neurol 79, 709-724 (1999).

55. Cobb, S.R., Buhl, E.H., Halasy, K., Paulsen, O. \& Somogyi, P. Synchronization of neuronal activity in hippocampus by individual GABAergic interneurons. Nature 378, 75-78 (1995).

56. Sipila, S.T., Huttu, K., Soltesz, I., Voipio, J. \& Kaila, K. Depolarizing GABA acts on intrinsically bursting pyramidal neurons to drive giant depolarizing potentials in the immature hippocampus. J Neurosci 25, 5280-5289 (2005).

57. Sipila, S.T., Huttu, K., Voipio, J. \& Kaila, K. Intrinsic bursting of immature CA3 pyramidal neurons and consequent giant depolarizing potentials are driven by a persistent $\mathrm{Na}+$ current and terminated by a slow $\mathrm{Ca} 2+$-activated $\mathrm{K}+$ current. Eur $J$ Neurosci 23, 2330-2338 (2006).

58. Khalilov, I., Minlebaev, M., Mukhtarov, M. \& Khazipov, R. Dynamic Changes from Depolarizing to Hyperpolarizing GABAergic Actions during Giant Depolarizing Potentials in the Neonatal Rat Hippocampus. J Neurosci 35, 12635-12642 (2015).

59. Kasyanov, A.M., Safiulina, V.F., Voronin, L.L. \& Cherubini, E. GABA-mediated giant depolarizing potentials as coincidence detectors for enhancing synaptic efficacy in the developing hippocampus. Proc Natl Acad Sci U S A 101, 39673972 (2004).

60. Leinekugel, X., Khalilov, I., Ben-Ari, Y. \& Khazipov, R. Giant depolarizing potentials: the septal pole of the hippocampus paces the activity of the developing intact septohippocampal complex in vitro. J Neurosci 18, 6349-6357 (1998).

61. Ben-Ari, Y., Gaiarsa, J.L., Tyzio, R. \& Khazipov, R. GABA: a pioneer transmitter that excites immature neurons and generates primitive oscillations. Physiol Rev 87, 1215-1284 (2007).

62. Roux, L., Hu, B., Eichler, R., Stark, E. \& Buzsaki, G. Sharp wave ripples during learning stabilize the hippocampal spatial map. Nat Neurosci 20, 845-853 (2017).

63. Oliva, A., Fernandez-Ruiz, A., Buzsaki, G. \& Berenyi, A. Role of Hippocampal CA2 Region in Triggering Sharp-Wave Ripples. Neuron 91, 1342-1355 (2016).

64. Hulse, B.K., Moreaux, L.C., Lubenov, E.V. \& Siapas, A.G. Membrane Potential Dynamics of CA1 Pyramidal Neurons during Hippocampal Ripples in Awake Mice. Neuron 89, 800-813 (2016).

65. Papatheodoropoulos, C. Patterned activation of hippocampal network (approximately $10 \mathrm{~Hz}$ ) during in vitro sharp wave-ripples. Neuroscience 168, 429442 (2010).

66. Sylantyev, S. \& Rusakov, D.A. Sub-millisecond ligand probing of cell receptors with multiple solution exchange. Nat Protoc 8, 1299-1306 (2013).

67. Madisen, L., et al. A toolbox of Cre-dependent optogenetic transgenic mice for light-induced activation and silencing. Nat Neurosci 15, 793-802 (2012).

68. Kopell, N., Borgers, C., Pervouchine, D., Malerba, P. \& Tort, A. Gamma and Theta Rhythms in Biophysical Models of Hippocampal Circuits. Spr Ser Comput Neuro 5, 423-457 (2010). 
69. Tort, A.B.L., Rotstein, H.G., Dugladze, T., Gloveli, T. \& Kopell, N.J. On the formation of gamma-coherent cell assemblies by oriens lacunosum-moleculare interneurons in the hippocampus. Proc. Natl. Acad. Sci. USA 104, 13490-13495 (2007).

70. Gloveli, T., et al. Orthogonal arrangement of rhythm-generating microcircuits in the hippocampus. Proc. Natl. Acad. Sci. USA 102, 13295-13300 (2005). 